\title{
Patrones arquitectónicos, clusters constructivos homogéneos y variabilidad en el estudio de edificios históricos. Aspectos técnico-formales de la vivienda rural en la provincia de La Rioja (Argentina) durante el período republicano
}

\author{
Architectural patterns, constructive homogeneous "clusters" and variability \\ in the study of historic buildings. Technical and formal aspects of rural dwelling \\ in La Rioja province (Argentina) throughout the Republican period
}

\author{
Guillermo Rolón \\ Instituto de Arte Americano e Investigaciones Estéticas «Mario J. Buschiazzo» \\ Universidad de Buenos Aires \\ e-mail: grolon@fadu.uba.ar
}

\section{RESUMEN}

El artículo presenta el procedimiento metodológico con el cual se analizaron las características de la arquitectura popular del ámbito rural en la provincia de La Rioja (Argentina) del período republicano (s. XIX a la actualidad). La aplicación integral de análisis estratigráficos y tipológicos, y el auxilio de técnicas analíticas multivariantes, permitió identificar y caracterizar los patrones arquitectónicos desarrollados. Se utilizó el concepto de patrón arquitectónico en el diseño metodológico, como alternativa al de tipo edilicio, para considerar y evaluar no sólo los aspectos estables (el cluster constructivo homogéneo o estructura interna del patrón), sino también aquellas características variables que se observaron en la edificación de las unidades domésticas consideradas.

Palabras clave: Vivienda vernácula; análisis multivariante; análisis factorial múltiple; vivienda doméstica; arquitectura de tierra.

\begin{abstract}
The paper presents the methodological procedure by which the characteristics of the traditional architecture in rural areas in La Rioja province (Argentina) of the Republican Period (from 19th century until nowadays) were analyzed. The comprehensive application of stratigraphic and typological analysis along with multivariate analytical techniques allowed the identification and characterization of the developed architectural patterns. The concept of architectural pattern was used within the methodological design, as an alternative to the building type, in order to consider and evaluate not only the stable aspects (the constructive and homogeneous "cluster" as internal structure of the pattern), but also those variable characteristics that were observed in the construction of the dwellings considered.
\end{abstract}

Key words: Vernacular architecture; multivariate analysis; multiple factor analysis; domestic dwelling; earthen architecture.

Recibido: 11 mayo 2014. Aceptado: 26 agosto 2014.

Cómo citar este artículo / Citation

Rolón, G.: "Patrones arquitectónicos, clusters constructivos homogéneos y variabilidad en el estudio de edificios históricos. Aspectos técnicoformales de la vivienda rural en la provincia de La Rioja (Argentina) durante el período republicano", Arqueología de la Arquitectura, 11 : e010. doi.org/10.3989/arq.arqt.2014.013

\section{Copyright}

(c) 2014 CSIC. Este es un artículo de acceso abierto distribuido bajo los términos de la licencia Creative Commons Attribution-Non Commercial (by-nc) Spain 3.0. 


\section{Introducción}

El presente trabajo expone un procedimiento metodológico para analizar los procesos complejos involucrados en la variabilidad de la producción de la arquitectura doméstica, entendiéndose como tal la interrelación que se establece entre diversos factores o variables, sean culturales, históricos, técnicos y/o ambientales. El análisis de la interrelación en el conjunto de variables y rasgos que suelen considerarse para caracterizar un objeto arquitectónico, y de aspectos sincrónicos y diacrónicos que están involucrados en estos procesos de variabilidad, constituye un campo temático escasamente abordado hasta el momento. Es posible que esta escasez radique no tanto en la carencia de interés en realizar dichos análisis como en la ausencia de técnicas analíticas y de una metodología adecuada para tal fin.

El abordaje metodológico que se presenta fue elaborado tomando en consideración algunos conceptos y avances actuales, como los estudios sobre patrones arquitectónicos y los clusters constructivos homogéneos, que vienen siendo desarrollados y discutidos en el último tiempo desde el ámbito de la Arqueología de la Arquitectura (Azkarate 2002; Plata 2003; Sánchez Zufiaurre 2007; García 2009; Azkarate y Solaun 2012).

Este estudio forma parte de una tesis doctoral concluida destinada a analizar los procesos históricos y tecnológicos que establecieron el marco referencial para la producción y reproducción de determinados patrones arquitectónicos de la vivienda rural popular en la región de los valles riojanos de Argentina durante el período republicano (Rolón 2013). El lapso temporal específico abarcó desde mediados del s. XIX, momento en el que se registra el establecimiento de gran parte del conjunto de viviendas analizadas en dicha investigación, hasta mediados del siglo siguiente, cuando la combinación de determinados procesos colaboran para la acelerada discontinuidad de los patrones arquitectónicos de la arquitectura doméstica.

Existe consenso en que, como parte de la cultura material, la arquitectura es un importante marco referencial para la interpretación de los contextos sociales (Mañana, Ayán y Blanco 2002; Ayán 2003 y 2014). Es evidente que su estudio posibilita abordar diversos aspectos involucrados en la construcción de los paisajes culturales tales como los procesos históricos y políticos, el análisis de los horizontes tecnológicos, la disponibilidad de recursos o características concernientes con niveles de significación simbólica de los espacios construidos (Azkarate y García
2004; Bermejo 2009; Caballero 2009) pero, fundamentalmente, permite dilucidar las complejas estrategias de organización social y territorial de una comunidad (Ayán 2014; Vigil-Escalera 2014). Con estos fines, una parte significativa de los estudios abordados desde la Arqueología de la Arquitectura sobre el patrimonio arquitectónico han tomado en consideración las expresiones más singulares (iglesias, fuertes, edificios públicos o cívicos singulares, etc.). En las últimas décadas, sin embargo, diversas investigaciones han profundizado y puesto en evidencia el importante reservorio de información que presenta la arquitectura menor, doméstica, popular (o como deba ser definida según el marco teórico que se adopte), para alcanzar objetivos semejantes (Azkarate y Solaun 2012; Azkarate 2013; Gutiérrez y Grau 2014).

\subsection{Tipología de la edificación}

Un marco teórico posible para efectuar este planteamiento y hacer comprensible la compleja trama interna en la que se desenvuelve la producción material de la arquitectura se encuentra en los estudios sobre la tipología de la edificación. Los estudios tipológicos, basados durante bastante tiempo en el concepto de «tipo edilicio» o «tipo de edificación» (Martín 1984; Caniggia y Maffei 1995), fueron muy recurrentes en la disciplina arquitectónica durante los últimos dos siglos. En la etapa presente, como resultado de una mayor complejidad analítica vinculada a los análisis sobre la relación dialéctica entre la unidad (el edificio) y el conjunto (la ciudad), y sobre los procesos históricos y continuos en la conformación de ambos, emergerán cuestiones que estuvieron ausentes en etapas precedentes (Güney 2007). En la actualidad, el objetivo se orienta a analizar diversos asuntos (como el uso del territorio, la conformación urbana, la estructuración del edificio, las técnicas constructivas, la elección de los materiales de construcción) vinculados al tema en cuestión y las interconexiones y procesos de retroalimentación entre cada uno de ellos a través de la historia, las formas de percepción de la realidad y el contexto socio-cultural. Es por ello que en la etapa contemporánea cobró relevancia una línea de pensamiento sistémico en la arquitectura que indaga sobre los aspectos más complejos de la conformación urbana (Rossi 1977; Caniggia y Maffei 1995; Diez 1996), cuestiones que, como señala Ardelean (2004), en la arqueología ya tenían una importante tradición, lo cual explica especialmente su actual cristalización en la Arqueología de la Arquitectura (Azkarate y García 2004; García 2009) y en la Arqueología del Paisaje (Criado 1999). 


\subsection{Patrón arquitectónico y cluster constructivo homogéneo}

Dentro del pensamiento sistémico acontece la introducción y definición del concepto de «Patrón»en arquitectura (Alexander, Ishikawa y Silverstein 1977; Alexander 1981)', en términos semejantes a los de «conciencia espontánea» de Caniggia y Maffei (1995), y en relación complementaria con la interpretación que realizan Hillier y Leaman (1974) de los conceptos «genotipo» $\mathrm{y}$ «fenotipo». El mayor aporte de estos autores, fundamentalmente de los últimos dos, radica en relevancia puesta sobre la estructura «subyacente» de un objeto arquitectónico; es decir, la red de relaciones que lo define como producto de la cultura material; red que se presenta relativamente constante entre objetos semejantes y permite establecer la clase tipológica a la que pertenecen. Esta postura comienza a cuestionar, en cierta medida, la construcción de tipos edilicios basada en unos pocos caracteres considerados diagnósticos (Sánchez Zufiaurre 2007), resultando sumamente importante porque pondrá el foco en determinar y describir la red de relaciones para la interpretación de los procesos en la conformación de la arquitectura y de los asentamientos humanos (Azkarate 2002).

De este modo, mediante el empleo del concepto de patrón en arquitectura es posible asumir la existencia de una red de relaciones, donde la variabilidad de esta red es en respuesta al contexto concreto de aplicación. Por lo tanto, definir el patrón implica realizar el intento de reconocer esta red de relaciones subyacente, permitiendo que la identificación tipológica emerja un tanto más objetiva respecto del operante, pero también más compleja.

Un aspecto actual del enfoque sistémico en el estudio tipológico de la edificación en el que se está profundizado procede de la taxonomía numérica y corresponde al análisis de conglomerados o análisis cluster (Mountford 1970; Sneath y Sokal 1973). La importancia de los

\footnotetext{
Alexander define al patrón como el dispositivo por el cual se describe un problema que ocurre de manera recurrente en nuestro entorno así como su solución y que, al ser compartido socialmente, se puede replicar adecuándolo a un contexto específico pero sin la necesidad de ser regenerado (Alexander, Ishikawa y Silverstein 1977: X). La particularidad del planteamiento realizado por el autor radica en que considera la existencia de un «lenguaje de patrones» prácticamente de la misma manera que lo es cualquier lenguaje. Para el autor, este lenguaje es el sistema genético de los objetos y surge de una relación dialéctica con el sustrato cultural y el entorno físico específico (Alexander 1981: 24). Sugiere, de este modo, que el lenguaje es un conjunto finito de reglas que posibilita generar una infinita variedad de elaboraciones diferentes (Alexander 1981: 158) y que organiza la presencia, disposición y articulación de los patrones entre sí.
}

análisis clusters radica en el aporte de una metodología de análisis multivariante para identificar los grados de semejanza entre elementos que conforman el continuo en una seriación. El factor que impulsó su empleo en la Arqueología de la Arquitectura fue la puesta en consideración de que para obtener clasificaciones satisfactorias no debía asignarse a juicio propio y a priori ninguna ponderación de las variables consideradas al analizar un conjunto de elementos (Sánchez Zufiaurre 2007). Al mismo tiempo, debido a que la cantidad de variables y de unidades de análisis suele ser numerosa en estos estudios, la implementación de métodos cuantitativos y multivariantes resultaba ciertamente insoslayable (por ejemplo, Van Dyke 1999; Pugh 2003).

La propuesta de este concepto en la Arqueología de la Arquitectura aconteció en la última década durante el proceso de restauración de la Catedral de Santa María de Vitoria-Gasteiz (Azkarate 2002). Mediante la proposición del concepto de «cluster constructivo homogéneo» sugerido por Azkarate, se evaluó la posibilidad de identificar e interpretar conjuntos de rasgos y variables que permitirían reflejar «...la homogeneidad formal que todo acto constructivo coetáneo conlleva intrínseco» (Azkarate 2002: 69). Debido a que este instrumento de análisis se encuentra en pleno proceso de evaluación, la cantidad de estudios efectivos no es numeroso, restringiéndose específicamente al análisis sobre la arquitectura religiosa medieval alavesa (Sánchez Zufiaurre 2007), a la conformación del complejo productivo de Salinas de Añana (Plata 2003) y a un estudio sobre la estructura urbana de la ciudad de Vitoria-Gasteiz en espera de salir a la luz (Azkarate 2011: 22).

\section{El estudio}

Este estudio incluyó el análisis de 31 viviendas rurales prospectadas entre 2008 y 2012, distribuidas en el sector noroccidental de la provincia de La Rioja (Fig. 1A), en la región de valles intermontanos: el valle oriental conocido como La Costa de Arauco, el valle central de Antinaco-Los Colorados, el valle occidental del Río Bermejo, y los valles occidentales menores del Río la Troya y el Bolsón de Jagüe. El recorte temporal específico quedó determinado entre la primera mitad del s. XIX, momento en que se establecen las viviendas más antiguas analizadas, hasta mediados del s. XX, cuando se produce el abandono de dichas viviendas y su coincidencia con la introducción de materiales convencionales industrializados en la edificación de la vivienda rural. 


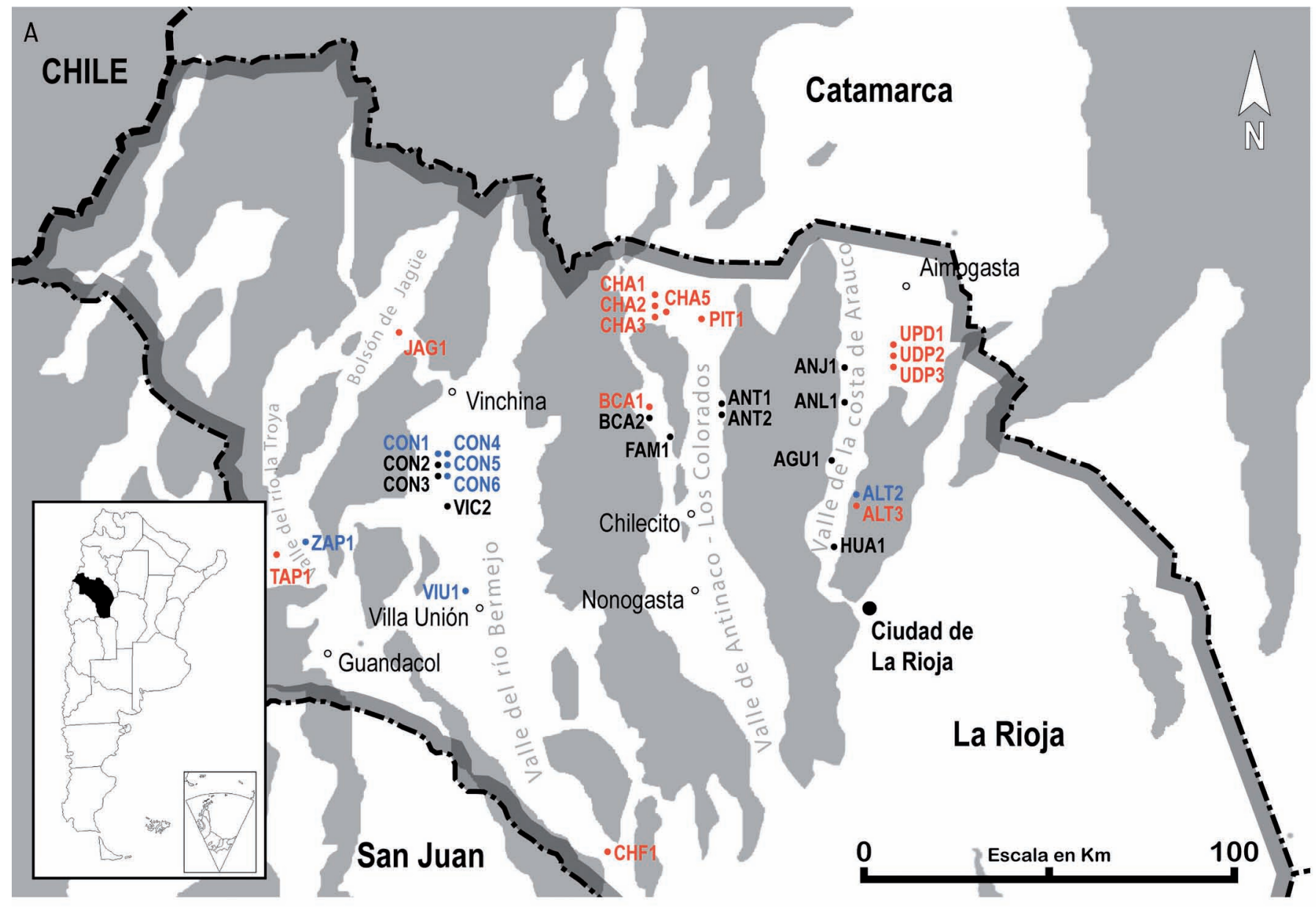

B

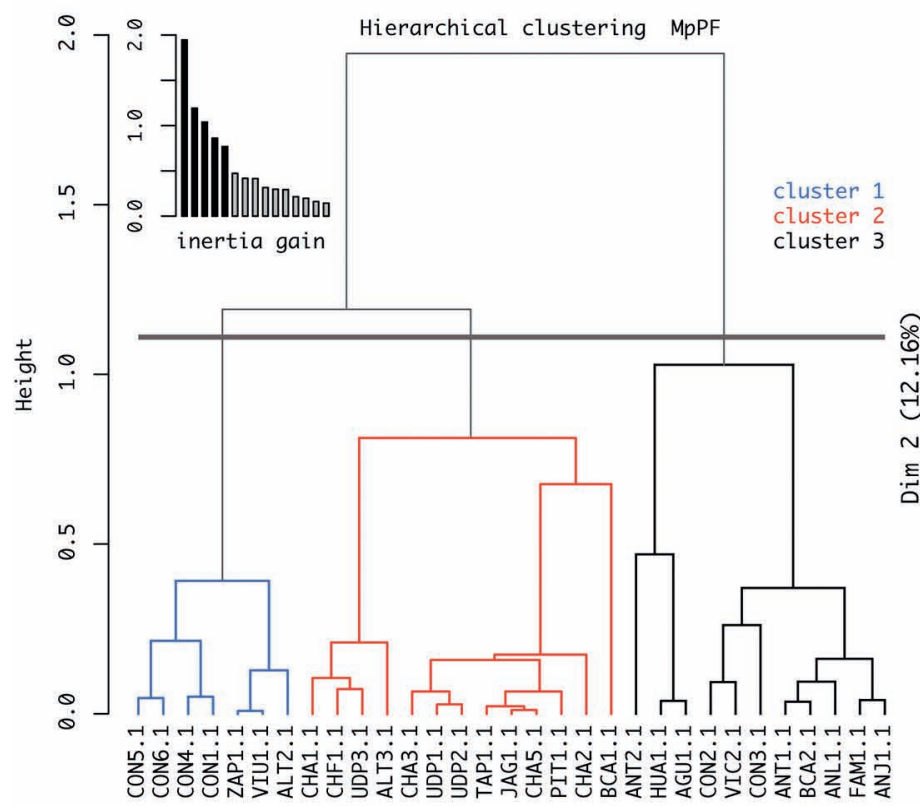

C

Factor map MpPF

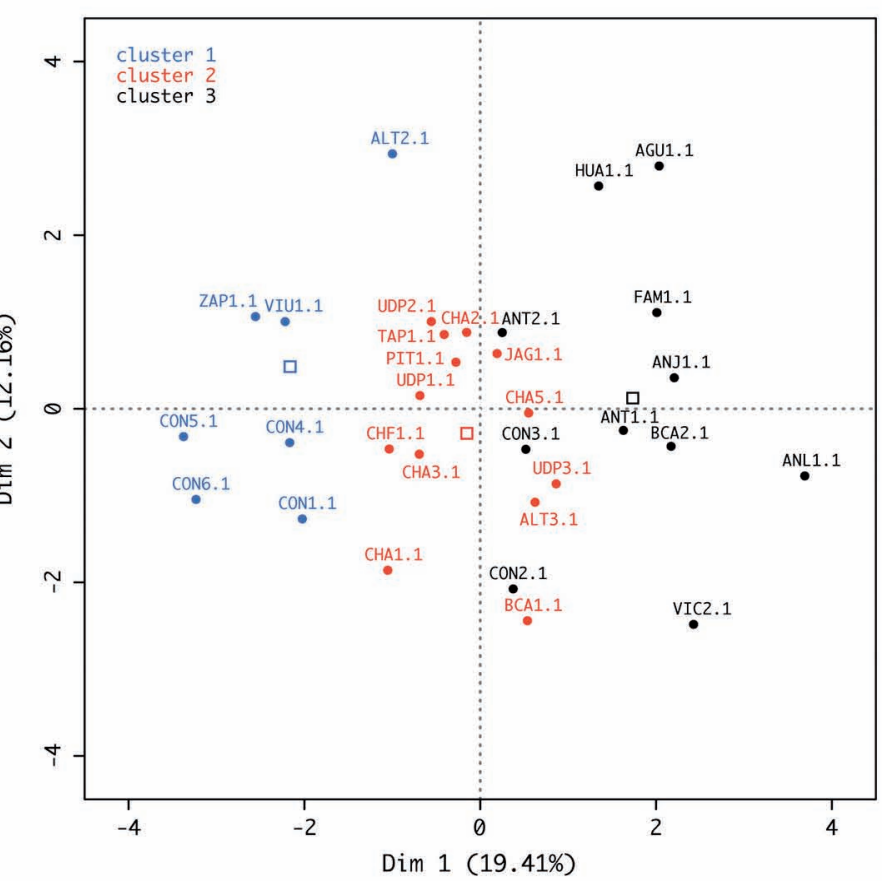

Figura 1. a) Distribución de las viviendas analizadas, los colores asignados corresponden a los clusters establecidos para MpPF; b) Cluster jerárquico de casos para MpPF, c) Representación de los clusters en el plano inducido por los dos primeros componentes principales. Los cuadrados indican los baricentros de los respectivos conglomerados. Fuente: Elaboración propia. 
Como se indicó en la introducción, el objetivo de este trabajo se remite a presentar el procedimiento metodológico utilizado para la identificación de los patrones arquitectónicos de las viviendas rurales para el área de estudio considerada y la caracterización de este último a partir de otros dos conceptos centrales: el cluster constructivo homogéneo y el margen de variabilidad del patrón arquitectónico. El primero resulta fundamental para poder definir un patrón arquitectónico, para entender cuáles son las variables y rasgos estructurales que determinan su cohesión interna y al mismo tiempo su diferenciación con respecto a otros patrones arquitectónicos. Esto se interpreta de tal manera porque el cluster constructivo homogéneo, como lo define Azkarate (2002: 69), constituye la red de relaciones entre variables y rasgos subyacentes que, al mantenerse relativamente constante entre diversos casos edilicios, les otorga un alto grado de semejanza entre sí. Por otra parte, y al no ser una red cerrada, las variables y rasgos menos estructurales pueden alterarse, $y$ con ello el caso concreto adaptarse al contexto específico de aplicación, teniendo un margen de variación, sin que supongan dejar de formar parte del patrón al que se asocian. Con este estudio no se plantea invalidar el uso del concepto de tipo en el estudio tipológico; el mismo, como bien señala Vargas (2013), continúa siendo una importante herramienta de análisis para determinados objetivos (por ejemplo, obtener dataciones de estratos).

\subsection{Metodología implementada}

El proceso de trabajo fue realizado en dos etapas tomando como referencia la metodología implementada por Sánchez Zufiaurre para el estudio de la arquitectura prerrománica alavesa (2007: 77): una primera etapa de análisis estratigráfico destinada a definir el proceso diacrónico de transformación de las viviendas y una etapa posterior correspondiente al abordaje tipológico en el que se evaluaron principalmente procesos sincrónicos.

La etapa de análisis estratigráfico consistió en determinar las distintas fases constructivas de cada vivienda para poder establecer el conjunto de casos de estudio (Fig. 2). Es decir, los casos de estudio no fueron las viviendas en sí mismas, sino las distintas fases constructivas que fue posible identificar en cada una de ellas. Esta definición de los casos de estudio tiene su explicación en que parte del interés que motivó el objetivo de la investigación consistió en examinar la incidencia de la variabilidad de rasgos (en cada vivienda y entre patrones) como parte del proceso diacrónico y dentro del lapso temporal considerado.

La segunda instancia de análisis correspondió al estudio tipológico y el auxilio de técnicas de análisis multivariante para la identificación y caracterización de los patrones arquitectónicos. La metodología llevada a cabo implicó como primer paso descomponer y
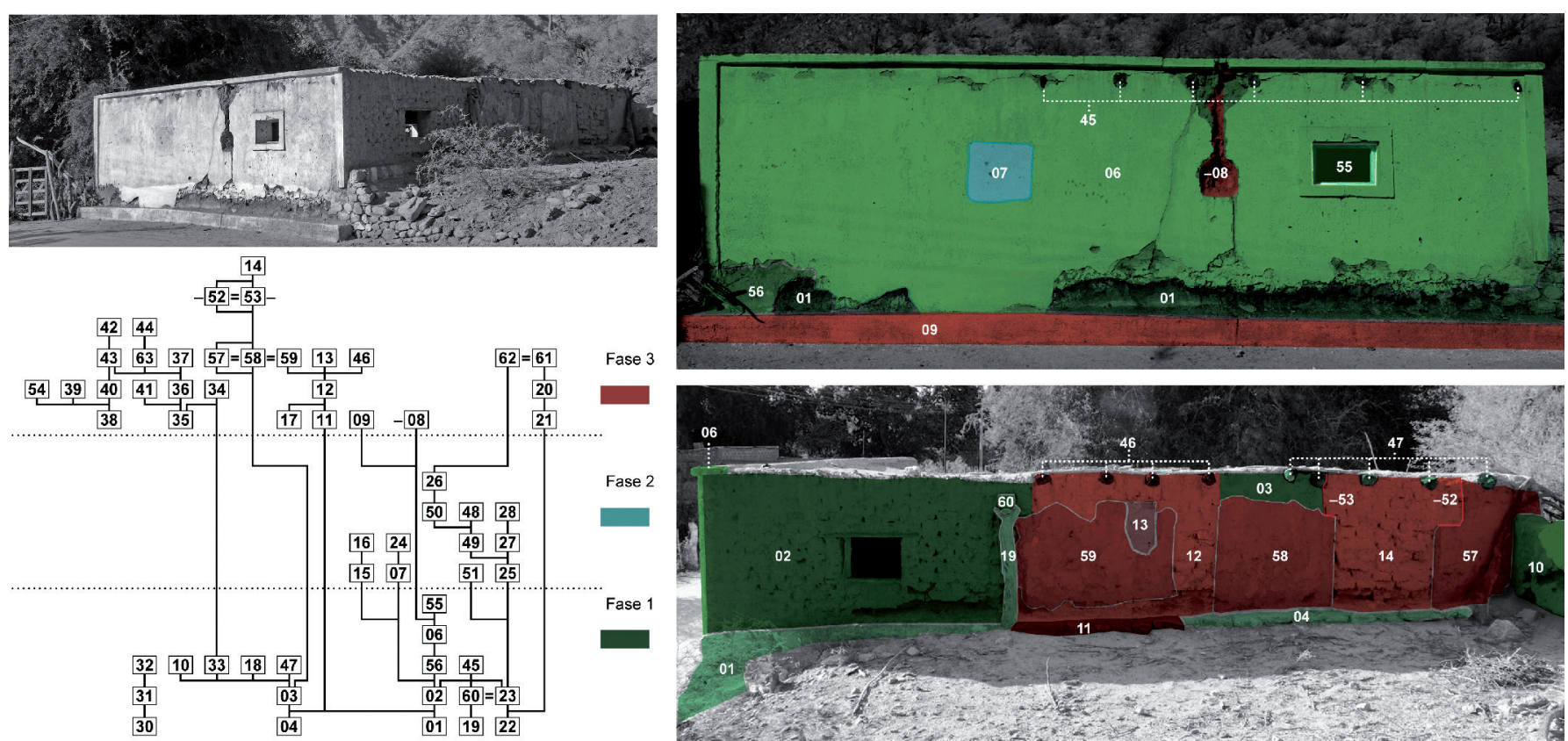

Figura 2. Ejemplo: análisis estratigráfico del caso ANT2 y determinación de las fases constructivas. Fuente: Elaboración propia y fotografías del archivo personal del autor. 
describir cada caso de estudio mediante un conjunto amplio de variables seleccionadas; luego, a través del empleo de técnicas específicas de análisis multivariante, determinar las articulaciones que subyacen entre las distintas variables consideradas, estableciendo con ello agrupamientos que sean estadísticamente significativos. Sánchez Zufiaurre comenta al respecto que «...una de las ideas fundamentales de este sistema de trabajo es la consideración de que los rasgos, de manera individual, no tienen valor diagnóstico. Son las interrelaciones entre variables las que permiten el establecimiento de categorías. [...] no se puede predecir (de antemano) qué rasgos o combinación de rasgos es pertinente o variante. Por ello se debe extraer la máxima cantidad de información de cada caso analizado, al menos en las fases preliminares de análisis, hasta el establecimiento de los rasgos que serán tomados en cuenta como constitutivos de similitud o diferencia entre "clusters"...» (Sánchez Zufiaurre 2007: 73-74). Es por ello que la instancia inicial de esta segunda parte se orientó a desarrollar un procedimiento metodológico para obtener dicho «establecimiento de rasgos» señalado.

\subsection{El proceso de trabajo}

En la selección de las viviendas analizadas se tuvieron en cuenta dos aspectos: por un lado considerar una cantidad equilibrada de edificios para los distintos valles que comprenden la región de estudio y por otro lado, dado el carácter exploratorio de la metodología multivariante implementada, se consideraron viviendas con particularidades, a priori singulares, para que funcionen en el análisis multivariante como elementos de control de la variabilidad ${ }^{2}$.

El procedimiento estratigráfico aplicado a este tipo de edificios fue explicitado en un artículo previo (Rolón y Rotondaro, 2010), motivo por el cual a continuación se describe exclusivamente el proceso realizado para el análisis tipológico. Este procedimiento incluyó las siguientes etapas de trabajo:

\footnotetext{
2 Con el fin de evaluar la eficacia del análisis estadístico multivariante se incluyeron deliberadamente casos de estudio con particularidades específicas para que se comportaran como casos marginales (en el sentido de los out layer). Estos casos se caracterizaron por el empleo exclusivo de entramados vegetales en toda la construcción (CON5.1) y casos de viviendas propios de poblados rurales envueltos en procesos de urbanización creciente (ANL1.1 y VIC2.1). También se consideró tomar como control las fases de viviendas en las que se observó el uso integral de materiales industrializados (UDP3.3 y CHA3.4).
}

\subsubsection{Definición y estados de las variables iniciales}

En primera instancia se seleccionaron rasgos que pudieran estar implicados tanto en la definición como en la variabilidad de los patrones arquitectónicos. Estos rasgos permitieron definir las variables iniciales de análisis, las cuales se organizaron en tres grandes grupos con diferente peso relativo entre ellos: de carácter tecnológico, de carácter morfológico/espacial y de localización. El peso relativo entre los grupos se aseguró a partir de la cantidad de variables que quedaron establecidas en cada uno de ellos. Es importante destacar que se reforzó el peso del grupo de variables tecnológicas para evitar, de esta manera, errores de valoración subjetiva que podrían derivarse de la interpretación morfológico/espacial y de los rasgos de emplazamiento; la intención es asegurar una observación con mayor grado de objetividad considerando los rasgos tecnológicos como los más fiables (Sánchez Zufiaurre 2007: 79).

\subsubsection{Construcción y denominación de las matrices}

La instancia de análisis tipológico por métodos de análisis multivariante tuvo dos objetivos: por un lado, identificar los patrones arquitectónicos a partir de un análisis cluster que estableciera la conglomeración de casos, y por otro lado, determinar la existencia de clusters constructivos homogéneos y el margen de variabilidad. Por tales motivos, una vez establecidas las variables de análisis, se procedió a la construcción de dos matrices de trabajo según la naturaleza de análisis que requería cada objetivo (en el primer caso se requiere la conglomeración de casos mientras que en el segundo de los rasgos de las variables). Las matrices fueron construidas con los mismos datos recogidos, pero el número de variables fue significativamente diferente para cada una. En la matriz utilizada para el análisis de patrones arquitectónicos (denominada en adelante $\mathrm{Mp}$ ) se consideraron 32 variables. Sin embargo, para la construcción de la matriz utilizada en el análisis de clusters constructivos homogéneos (denominada en adelante $\mathrm{Mc}$ ), las variables (cuantitativas y cualitativas) fueron transformadas en variables cualitativas dicotómicas de la forma «presencia / ausencia». Para que esto fuera posible, gran parte de los estados de variables de Mp fueron transformados en variables nuevas para Mc. Esto último provocó que se pasara a 130 variables en Mc. En el caso de variables cuantitativas continuas se utilizaron intervalos de clase. 
Por otra parte, con el fin de analizar la variabilidad de los patrones arquitectónicos y de los clusters constructivos homogéneos en términos diacrónicos se efectuó un procedimiento que consistió en realizar la comparación entre el conjunto formado por la totalidad de casos de estudio $(\mathrm{G})$ y el subconjunto integrado únicamente por las primeras fases constructivas de cada vivienda analizada $(\mathrm{PF})$. Para llevar a cabo este procedimiento se realizaron matrices complementarias, tanto en $\mathrm{Mp}$ como en $\mathrm{Mc}$, considerando sólo las primeras fases de las viviendas analizadas. De este modo se establecieron cuatro matrices de trabajo: $\mathrm{MpG}, \mathrm{MpPF}, \mathrm{McG}$ y McPF.

\subsubsection{Análisis de patrones arquitectónicos}

Para realizar el clustring con el fin de identificar los patrones arquitectónicos se utilizó el programa estadístico ' $\mathrm{R}$ ' ${ }^{3}$ aprovechando varias de las particularidades que presenta: a) estudio de variables estructuradas en grupos; b) Análisis conjunto de variables cualitativas y cuantitativas como elementos activos; c) consideración de variables suplementarias, es decir, variables que no entran en el cálculo de la distancia entre casos pero su representación posterior permite colaborar en el proceso de interpretación de los resultados; d) salidas gráficas de lectura complementaria para la instancia de interpretación de resultados (Pagès 2004; Lê, Josse y Husson 2008; Husson, Josse y Pagès 2010). En relación al inciso a, para el presente estudio se establecieron los siguientes grupos de variables (Fig. 3): Muros (variables 1 a 8), Techos (9 a 11), Aberturas (12 a 16), Implantación (17 a 19), Orden (20 a 22), Superficies (23 a 27), Emplazamiento (28 y 29) y Posición (variables 30 a 32).

Un paso preliminar y sumamente importante fue evaluar las variables mediante un análisis de componentes principales (salvo las variables de carácter cualitativo que no pueden ser analizadas mediante este procedimiento). Con este análisis se determinó el aporte individual de cada una de ellas (inercia) a la variabilidad del modelo y la existencia de variables pertenecientes a un mismo grupo que estuvieran correlacionadas ${ }^{4}$.

\footnotetext{
3 Development Core Team (2009). R: A language and environment for statistical computing. R Foundation for Statistical Computing, Vienna, Austria. ISBN 3-900051-07-0. [En línea] http://www.R-project.org [consultado el 10/09/2014], paquete empleado: FactoMineR avalado por CRAN (Lê, Josse y Husson 2008).

4 El análisis multivariante requiere de variables independientes para describir adecuadamente la variabilidad. En este caso, sobre variables de un mismo grupo que se observaron correlacionadas se obtuvo el coeficiente de correlación y se realizó la prueba de significación de Pearson para confirmar dicha la correlación.
}

De modo tal que, con el fin de reducir el ruido en la representación de la variabilidad general que pudieren introducir aquellas con escasa inercia (se consideraron de escasa inercia aquellas variables proyectadas en el primer plano factorial que no superaron el círculo del primer tercio del diámetro total de la representación) o con información redundante por estar correlacionadas entre sí, fueron descartadas (Fig. 4A). Este análisis se realizó para las matrices MpPF y MpG.

A continuación se procedió a realizar un Análisis Factorial Múltiple (AFM) sobre las variables activas y suplementarias finales. Los casos considerados en la matriz se agruparon (clustering) por el método de la media (Average linkage) y para la medida de asociación entre datos se consideró como algoritmo de cálculo la distancia euclidiana. A partir del resultado obtenido se denominaron: a) «casos marginales» a aquellos casos extremos pero no alejados de otros casos y dentro de un cluster; b) «casos singulares» a aquellos que se ubican aislados de cualquier otro, aún formando parte de un cluster.

\subsubsection{Análisis de clusters constructivos homogéneos}

Para caracterizar los patrones arquitectónicos se realizó un análisis multivariante, en este caso, sobre el conjunto de variables seleccionadas originalmente (sin considerar en forma activa las variables de Posición). El objetivo de este análisis fue determinar qué conjunto de variables, o más específicamente, qué conjunto de estados de las variables son concurrentes para la mayor parte de los casos de estudio; es decir, qué estados de variables se articulan entre sí en un edificio y esta articulación aparece, además, de forma reiterada en otras construcciones. Para este análisis se utilizó el programa estadístico PAST ver. 2.17 (Hammer, Harper y Ryan 2001). Los casos se agruparon por el método del «vecino más cercano» (neighbour joining), en tanto la medida de similitud entre ellos se estimó con el coeficiente de similitud de Jaccard. Se utilizó este coeficiente porque permite el procesamiento de matrices que contengan datos binarios y no considera las concurrencias negativas. Este método, al igual que el aplicado para la determinación de patrones, consiste en dos procesos, en primera instancia establece los grados de similitud entre los individuos de la población (establece el coeficiente de Jaccard para cada par de casos) y luego procede al armado del diagrama de agrupamiento según el método elegido. 


\begin{tabular}{|c|c|c|c|c|c|c|c|}
\hline DENOM. & VARIABLE & ESTADOS & ID & DENOM. & VARIABLE & ESTADOS & ID \\
\hline \multirow{4}{*}{ TMURO } & 1.Técnica & Quincha & $1 . Q$ & APUERTA & 16. Ancho & $x \leq 80$ & 16.80 \\
\hline & constructiva & Albañilería (adobe) & $1 . \mathrm{A}$ & & promedio & 90 & 16.90 \\
\hline & & Alabñilería (bloque) & 1.B & & puertas & 100 & 16.100 \\
\hline & & Albañilería (piedra) & 1.P & & & 110 & 16.110 \\
\hline \multirow[t]{6}{*}{ TAPAREJO } & 2.Tipo de & Soga & 2.5 & & & $120 \leq x$ & 16.120 \\
\hline & aparejo & Tizón & 2.T & Var. & 17.Forma de & Nuclear & $17 . \mathrm{NUC}$ \\
\hline & muro & Compuesto & 2. $C$ & Cual. & implantación & Adición separada & 17. SEP \\
\hline & & Inglés & 2.I & & & Adición yuxtapuesta & 17. YUX \\
\hline & & Ordinario & 2.0 & & & Adición Combinada & 17. COM \\
\hline & & Enripiado/concertado & 2.E & Var. & 18.0rientación & Este & $18 . \mathrm{E}$ \\
\hline \multirow[t]{4}{*}{ TSOBREC } & 3.Técnica & Sin sobrecimiento & 3.NS & Cual. & ingreso & Norte & $18 . \mathrm{N}$ \\
\hline & constructiva & Piedra ordinaria & 3.MO & & principal & Oeste & 18.0 \\
\hline & sobrecimiento & Piedra enripiada/concertada & 3.ME & & & Sur & 18.5 \\
\hline & & Hormigón simple & 3.HS & Var. & 19.0rientación & Este & $19 . \mathrm{E}$ \\
\hline \multirow[t]{4}{*}{ ESPMUROA } & 4.Espesor & $x \leq 20[\mathrm{~cm}]$ & 4.20 & Cual. & Galería/Patio & Norte & $19 . \mathrm{N}$ \\
\hline & muros & $21-40$ & 4.40 & & & Oeste & 19.0 \\
\hline & principales & $41-60$ & 4.60 & & & Sur & $19 . \mathrm{S}$ \\
\hline & & $61-80$ & 4.80 & $\overline{O G E O}$ & 20.0rganizaciór & Centralizada & $20 . \mathrm{C}$ \\
\hline \multirow[t]{5}{*}{ HMURO } & 6.Altura & $150-200[\mathrm{~cm}]$ & 6.200 & & espacial & Centralizadas agrupada & 20.CA \\
\hline & promedio & $201-250$ & 6.250 & & geométrica & Agrupada & $20 . \mathrm{A}$ \\
\hline & muro & $251-300$ & 6.300 & & & Lineal agrupada & 20.LA \\
\hline & & $301-350$ & 6.350 & & & Lineal & $20 . \mathrm{L}$ \\
\hline & & $351-400$ & 6.400 & FINGRESO & 21. Forma & Por galería & 21.1 \\
\hline \multirow[t]{4}{*}{ HSOBREC } & 7.Altura & Escasa $(x \leq 20)[\mathrm{cm}]$ & $7 . E$ & & de ingreso & Por patio int. - galería & 21.2 \\
\hline & sobrecimiento & Pequeña $(21-40)$ & 7.P & & & Por patio int. & 21.3 \\
\hline & & Media $\quad(41-60)$ & 7.M & & & Por patio int. - frente & 21.4 \\
\hline & & Grande $(60<x)$ & $7 . G$ & & & Por frente & 21.5 \\
\hline \multirow{5}{*}{ PAISLADO } & 8.Elemento & Horcón & $8 . \mathrm{H}$ & GAMMA & 22.Patrón & Tipo 1 & 22.1 \\
\hline & portante & Pilar adobe s/basa & 8.SB & & permeabilidad & Tipo 2 & 22.2 \\
\hline & aislado & Pilar adobe c/basa & 8.CB & & & Tipo 3 & 22.3 \\
\hline & & Pilar piedra c/basa & 8.P & & & Tipo 4 & 22.4 \\
\hline & & Col. $\mathrm{H}^{\circ} \mathrm{A}^{\circ} /$ Pilar ladrillo & 8.H/P & & & Tipo 5 & 22.5 \\
\hline \multirow{8}{*}{ TCUBIERTA } & 9.Tipo & Subtipo A ó B (liviana) & 9.1 & & & Tipo 6 & 22.6 \\
\hline & cubierta & Subtipo D (liviana) & 9.2 & & & Tipo 7 & 22.7 \\
\hline & en techo & Subtipo C ó E (liviana) & 9.3 & & & Tipo 8 & 22.8 \\
\hline & & Subtipo F ó G (pesada) & 9.4 & & & Tipo 9 & 22.9 \\
\hline & & Subtipo H ó I (pesada) & 9.5 & IEPRIV & 23.Indice & Muy bajo $(x \leq 20)[\%]$ & 23.MB \\
\hline & & Subtipo J (pesada) & 9.6 & & de espacios & Bajo $\quad(20-40)$ & $23 . \mathrm{B}$ \\
\hline & & Subtipo K ó L (pesada) & 9.7 & & privados & $(40-60)$ & 23. M \\
\hline & & Losa armada o vigetas & 9.8 & & & $(60-80)$ & 23. A \\
\hline \multirow{4}{*}{ PENDTECHO } & 10.Pendiente & Muy escasa $(x \leq 5)[\%]$ & 10.ME & & & Muy al to $(80<x)$ & 23.MA \\
\hline & en techo & Escasa $\quad(5-10)$ & $10 . \mathrm{E}$ & IVENTILA & 24.Índice & Muy bajo $(x \leq 10)[\%]$ & $24 . \mathrm{MB}$ \\
\hline & principal & Muy suave $(10-15)$ & 10.MS & & de ventilación & Bajo $\quad(10-20)$ & $24 . B$ \\
\hline & & Suave $\quad(15<x)$ & 10.5 & & & $(20-30)$ & 24.M \\
\hline \multirow[t]{3}{*}{ SEPTECHO } & 11.Separación & Pequeña $(x \leq 50)[\mathrm{cm}]$ & $11 . P$ & & & Alto $\quad(30<x)$ & $24 . A$ \\
\hline & estructura & Mediana $(50-75)$ & 11.M & SCONSTR & 25.Superficie & Muy pequeña $(x \leq 40)\left[\mathrm{m}^{2}\right]$ & 25.MP \\
\hline & de techo & Grande $\quad(75<x)$ & $11 . \mathrm{G}$ & & total & Pequeña $\quad(40-80)$ & $25 . P$ \\
\hline \multirow[t]{6}{*}{ TVENTANA } & 12.Ventana & Sin ventana & 12.0 & & construida & $(80-120)$ & $25 . M$ \\
\hline & & Vano Triangular & 12.1 & & & $(120-160)$ & $25 . G$ \\
\hline & & Vano Rectangular & 12.2 & & & Muy grande $(160<x)$ & 25.MG \\
\hline & & Hoja madera s/derrame & 12.3 & SGALERIA & 26.Superficie & Pequeña $\quad(x \leq 20)\left[m^{2}\right]$ & $26 . P$ \\
\hline & & Hoja madera c/derrame & 12.4 & & de galería & $(20-40)$ & 26.M \\
\hline & & Hoja chapa s/derrame & 12.5 & & & $(40-60)$ & $26 . \mathrm{G}$ \\
\hline \multirow[t]{3}{*}{ HANTEP } & 13.Altura & Baja $(x \leq 75)[\mathrm{cm}]$ & $13 . \mathrm{B}$ & & & Muy grande $(60<x)$ & 26.MG \\
\hline & promedio & Media $(75-110)$ & 13.M & SHUMEDAS & 27.Superficies & Muy pequeña $(x \leq 5)\left[\mathrm{m}^{2}\right]$ & 27.MP \\
\hline & antepechos & Alta $(110<x)$ & 13.A & & húmedas & Pequeña $\quad(5-10)$ & $27 . P$ \\
\hline SVENTANA & 14.Superficie & Pequeña $(\mathrm{x} \leq 0,40)\left[\mathrm{m}^{2}\right]$ & 14.P & & & Mediana & 27.M \\
\hline & promedio & Media $\quad(0,40-1,00)$ & $14 . \mathrm{M}$ & & & Grande $\quad(15<x)$ & 27.6 \\
\hline & ventanas & Grande $\quad(1,00<x)$ & $14 . \mathrm{G}$ & ASENTAM & 28.Tipo de & Rural aislado & $28 . R$ \\
\hline TPUERTA & 15.Puerta & Vano & 15.1 & & asentamiento & Poblado rural & 28.PR \\
\hline & & Hoja madera s/derrame & 15.2 & & & Poblado rural c/rasgos urb. & 28.PRU \\
\hline & & Hoja madera c/derrame & 15.3 & EMPLAZAM & & Barreal & 29.1 \\
\hline & & Hoja chapa s/derrame & 15.4 & & Emplazami ento & Barranca & 29.2 \\
\hline & & & & & natural & Llano & 29.3 \\
\hline & & & & & & Faldeo & 29.4 \\
\hline & & & & & & Ladera & 29.5 \\
\hline
\end{tabular}

Figura 3. Variables consideradas, denominación simplificada y estados posibles de las mismas. Fuente: Elaboración propia. 

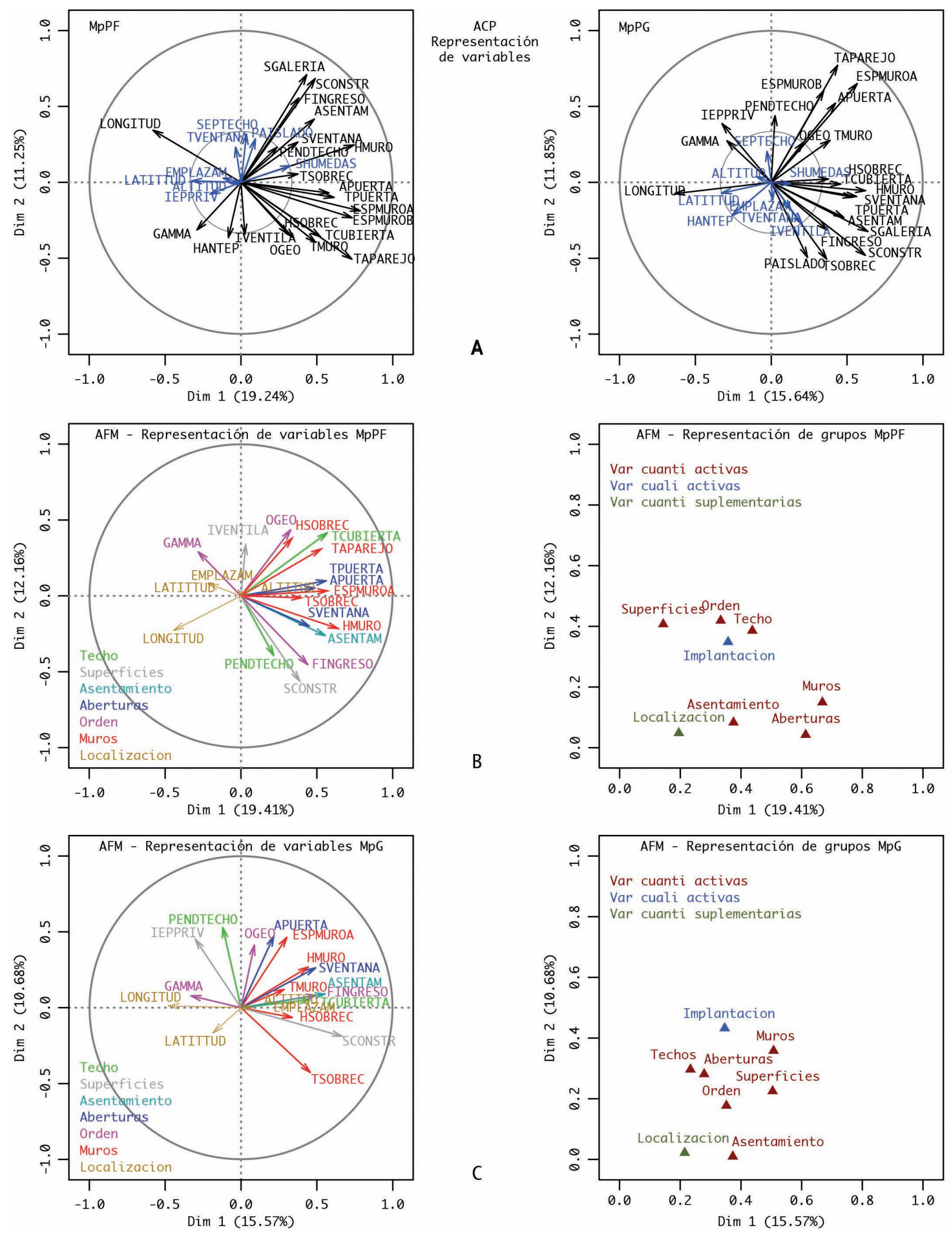

Figura 4. a) Representación de las variables para MpPF y MpG (luego del ACP). Las siglas corresponden a la denominación de las variables escogidas. En azul las variables consideradas de menor aporte tomándose como referencia un círculo de un tercio del diámetro total; b) Representación de las variables finales (izquierda) y de los grupos de variables (derecha) en el primer plano factorial luego del AFM para MpPF; c) Representación de las variables finales (izquierda) y de los grupos de variables (derecha) en el primer plano factorial luego del AFM para MpG. Fuente: Elaboración propia. 


\section{Resultados}

Mediante el análisis estratigráfico aplicado a las 31 viviendas analizadas fueron identificados un conjunto total de 75 fases constructivas (Fig. 5). A continuación, y luego de descartar preliminarmente algunas de las variables según los criterios considerados, se reservaron para MpPF 19 variables activas y 4 variables suplementarias. Para MpG fueron 18 las variables activas y 4 las variables suplementarias. Las variables suplementarias correspondieron específicamente a las de localización (altitud, latitud, longitud y emplazamiento). El paso siguiente consistió en realizar un clustering por medio de un AFM para las matrices MpPF y MpG.

\subsection{Patrones arquitectónicos}

\subsubsection{Interpretación de resultados para MpPF}

El cluster jerárquico de casos (dendograma) para MpPF se construyó considerando los primeros seis componentes del AFM. Con ello se logró explicar alrededor del
$65,33 \%$ de la variabilidad total del modelo y se observó la existencia de tres agrupamientos posibles (Fig. 1B). En la Figura 1A se representa la distribución territorial de los casos según las aglomeraciones obtenidas. Puede observarse que los clusters azul y negro tienden a agrupar a los casos que se ubican hacia los sectores centrales de los distintos valles, en tanto que el cluster naranja agrupa a los casos que se ubican en los sectores periféricos de los mismos y es a la vez el conglomerado más numeroso. A pesar de que para el AFM las variables de localización han sido incluidas como variables suplementarias (de este modo, sin incidencia en el proceso de cálculo), la presencia de grupos de casos tales como [ANT1 - ANT2], [CON2 - CON3 - VIC2], [CON1 CON4 - CON5 - CON6], [UPD1 - UPD2 - UPD3] y [CHA1 - CHA2 - CHA3 - CHA5 - PIT1] dan la pauta de que la proximidad espacial constituye un factor significativo de aglomeración.

Los clusters fueron representados empleando como ejes de representación los dos primeros componentes principales del AFM (Fig. 1C). Considerando la

\begin{tabular}{|c|c|c|c|c|c|c|c|c|c|}
\hline \multirow{2}{*}{$\begin{array}{l}\text { Poblado } \\
\text { o paraje }\end{array}$} & \multirow{2}{*}{\multicolumn{2}{|c|}{$\begin{array}{l}\text { ID } \\
\text { Viv. }\end{array}$}} & \multicolumn{5}{|c|}{$\begin{array}{l}\text { ID } \\
\text { Casos de estudio }\end{array}$} & \multicolumn{2}{|c|}{$\begin{array}{l}\text { Coordenadas de } \\
\text { ubicación }\end{array}$} \\
\hline & & & Fase 1 & Fase 2 & Fase 3 & Fase 4 & Fase 5 & Latitud & Longitud \\
\hline Agua blanca & AGU & 1 & 1.1 & 1.2 & 1.3 & & & $28^{\circ} 57,825^{\prime} \mathrm{s}$ & $67^{\circ} 00,100^{\prime} 0$ \\
\hline Los Altos & ALT & 2 & 2.1 & 2.2 & 2.3 & & & $29^{\circ} 07,845^{\prime} \mathrm{s}$ & $66^{\circ} 59,960^{\prime} 0$ \\
\hline Los Altos & ALT & 3 & 3.1 & & & & & $29^{\circ} 07,190^{\prime} \mathrm{s}$ & $66^{\circ} 58,560^{\prime} 0$ \\
\hline Anjullón & ANJ & 1 & 1.1 & 1.2 & 1.3 & 1.4 & & $28^{\circ} 42,775^{\prime} \mathrm{s}$ & $66^{\circ} 55,850^{\prime} 0$ \\
\hline Anillaco & ANL & 1 & 1.1 & 1.2 & 1.3 & 1.4 & 1.5 & $28^{\circ} 48,725^{\prime} \mathrm{s}$ & $66^{\circ} 56,120^{\prime} 0$ \\
\hline Antinaco & ANT & 1 & 1.1 & 1.2 & 1.3 & & & $28^{\circ} 49,400^{\prime} \mathrm{S}$ & $67^{\circ} 18,810^{\prime} 0$ \\
\hline Antinaco & ANT & 2 & 2.1 & 2.2 & 2.3 & & & $28^{\circ} 49,145^{\prime} \mathrm{S}$ & $67^{\circ} 18,945^{\prime} 0$ \\
\hline Bajo Carrizal & $\mathrm{BCA}$ & 1 & 1.1 & 1.2 & & & & $28^{\circ} 53,585^{\prime} \mathrm{s}$ & $67^{\circ} 33,270^{\prime} 0$ \\
\hline Bajo Carrizal & $\mathrm{BCA}$ & 2 & 2.1 & 2.2 & 2.3 & 2.4 & & $28^{\circ} 53,640^{\prime} \mathrm{s}$ & $67^{\circ} 33,210^{\prime} 0$ \\
\hline Chañarmuyo & $\mathrm{CHA}$ & 1 & 1.1 & & & & & $28^{\circ} 35,900^{\prime} \mathrm{s}$ & $67^{\circ} 33,980^{\prime} 0$ \\
\hline Chañarmuyo & $\mathrm{CHA}$ & 2 & 2.1 & 2.2 & & & & $28^{\circ} 35,990^{\prime} \mathrm{s}$ & $67^{\circ} 34,090^{\prime} 0$ \\
\hline Chañarmuyo & $\mathrm{CHA}$ & 3 & 3.1 & 3.2 & 3.3 & 3.4 & & $28^{\circ} 36,070^{\prime} \mathrm{s}$ & $67^{\circ} 34,145^{\prime} 0$ \\
\hline Chañarmuyo & $\mathrm{CHA}$ & 5 & 5.1 & & & & & $28^{\circ} 36,340^{\prime} \mathrm{S}$ & $67^{\circ} 34,400^{\prime} 0$ \\
\hline El Chiflón & $\mathrm{CHF}$ & 1 & 1.1 & 1.2 & & & & $30^{\circ} 12,690^{\prime} \mathrm{s}$ & $67^{\circ} 33,280^{\prime} 0$ \\
\hline El Condado & $\mathrm{CON}$ & 1 & 1.1 & 1.2 & & & & $28^{\circ} 59,270^{\prime} \mathrm{s}$ & $68^{\circ} 13,850^{\prime} 0$ \\
\hline El Condado & $\mathrm{CON}$ & 2 & 2.1 & 2.2 & & & & $28^{\circ} 59,190^{\prime} \mathrm{s}$ & $68^{\circ} 13,815^{\prime} 0$ \\
\hline El Condado & CON & 3 & 3.1 & 3.2 & 3.3 & & & $28^{\circ} 59,120^{\prime} \mathrm{S}$ & $68^{\circ} 13,750^{\prime} 0$ \\
\hline El Condado & $\mathrm{CON}$ & 4 & 4.1 & 4.2 & 4.3 & & & $28^{\circ} 58,925^{\prime} \mathrm{s}$ & $68^{\circ} 13,480^{\prime} 0$ \\
\hline El Condado & CON & 5 & 5.1 & & & & & $28^{\circ} 58,975^{\prime} \mathrm{s}$ & $68^{\circ} 13,675^{\prime} 0$ \\
\hline El Condado & $\mathrm{CON}$ & 6 & 6.1 & 6.2 & & & & $28^{\circ} 58,940^{\prime} \mathrm{S}$ & $68^{\circ} 13,745^{\prime} 0$ \\
\hline Famatina & FAM & 1 & 1.1 & 1.2 & 1.3 & & & $28^{\circ} 58,135^{\prime} \mathrm{s}$ & $67^{\circ} 30,685^{\prime} 0$ \\
\hline Huaco & HUA & 1 & 1.1 & 1.2 & & & & $29^{\circ} 12,250^{\prime} \mathrm{S}$ & $67^{\circ} 02,480^{\prime} 0$ \\
\hline El Jagüe & JAG & 1 & 1.1 & 1.2 & & & & $28^{\circ} 39,250^{\prime} \mathrm{S}$ & $68^{\circ} 20,705^{\prime} 0$ \\
\hline Pituil & PIT & 1 & 1.1 & 1.2 & 1.3 & & & $28^{\circ} 34,280^{\prime} \mathrm{S}$ & $67^{\circ} 26,960^{\prime} 0$ \\
\hline Las tapias & TAP & 1 & 1.1 & 1.2 & 1.3 & & & $29^{\circ} 16,885^{\prime} \mathrm{S}$ & $68^{\circ} 44,565^{\prime} 0$ \\
\hline Udpinango & UDP & 1 & 1.1 & 1.2 & & & & $28^{\circ} 40,390^{\prime} \mathrm{s}$ & $66^{\circ} 49,140^{\prime} 0$ \\
\hline Udpinango & UDP & 2 & 2.1 & 2.2 & & & & $28^{\circ} 40,490^{\prime} \mathrm{S}$ & $66^{\circ} 48,800^{\prime} 0$ \\
\hline Udpinango & UDP & 3 & 3.1 & 3.2 & 3.3 & & & $28^{\circ} 40,510^{\prime} \mathrm{S}$ & $66^{\circ} 48,770^{\prime} 0$ \\
\hline Villa Castelli & VIC & 2 & 2.1 & & & & & $29^{\circ} 01,650^{\prime} \mathrm{s}$ & $68^{\circ} 13,990^{\prime} 0$ \\
\hline Villa Unión & VIU & 1 & 1.1 & 1.2 & & & & $29^{\circ} 16,140^{\prime} \mathrm{S}$ & $68^{\circ} 14,300^{\prime} 0$ \\
\hline Zapallar & ZAP & 1 & 1.1 & & & & & $29^{\circ} 17,430^{\prime} \mathrm{S}$ & $68^{\circ} 40,720^{\prime} 0$ \\
\hline
\end{tabular}

Figura 5. Viviendas analizadas y casos de estudio establecidos. Fuente: Elaboración propia. 
distribución de los baricentros de cada uno, su distribución se observa más sensible al primer eje factorial. En cambio, la distribución de los casos agrupados en cada cluster es más sensible a la variabilidad registrada por el segundo eje factorial. La interpretación de estos resultados permite sugerir que los patrones arquitectónicos en MpPF tienden a diferenciarse entre sí fundamentalmente por aspectos ligados a resoluciones tecnológicas y luego, internamente dentro de cada cluster, son los aspectos morfológicos los que entran en juego para explicar la variabilidad de los casos de estudios. Esto surge de la lectura complementaria entre los gráficos de las Figuras 1C y 4B donde se observa una correlación de los grupos de variables «Muros» $\mathrm{y}$ «Aberturas» con la primera dimensión y los de los grupos de variables «Orden», «Implantación» $\mathrm{y}$ «Superficies» con la segunda dimensión.

Por otra parte, al examinar los casos tomados como control, se confirmó la posición marginal de CON5.1 en la distribución. Asimismo, se observaron como casos singulares ALT2.1 (lo cual se explica por su valores atípicos de superficie construida y altura de muros en extremo bajos), ANL1.1 y VIC2.1, (Fig. 1C). Estos dos últimos corresponden a casos característicos de arquitectura vernácula urbana en tierra según la clasificación propuesta por Armellini, Cóppola, Iglesias y Rosso (1970) y además presentan valores de superficie muy elevados para una primera fase constructiva.

\subsubsection{Características de los patrones arquitectónicos vigentes durante la segunda mitad del s. XIX}

Para no abrumar con los detalles que permitieron caracterizar puntualmente a cada uno de los patrones arquitectónicos observados sólo se presentará una breve comparación entre los mismos. El primer aspecto a destacar es la correlación que se presenta entre el nivel de sofisticación de las técnicas constructivas empleadas en cada patrón arquitectónico para resolver los edificios y el tipo de asentamiento con el que se identifican. Aquellas viviendas que se encuentran preferentemente en asentamientos rurales aislados tendieron a conglomerarse en el primer patrón arquitectónico (cluster azul) haciendo uso de las resoluciones tecnológicas más elementales y ciertamente precarias en comparación con los otros patrones arquitectónicos. Es decir, la relación que se establece principalmente entre el uso de delgados muros de albañilería de adobe (por presentar aparejos a soga o entramados de «quincha $»^{5}$ ) y de escasa altura; el empleo de cubiertas de «torta liviana $»^{6}$ con muy escasa pendiente y la carencia de «sobrecimientos». En contraste, las viviendas que se emplazan en poblados netamente rurales o bien con algunos rasgos urbanos ${ }^{7}$ implementan resoluciones tecnológicas un tanto más sólidas y perdurables donde se observa el empleo de cubiertas de tipo pesadas y paredes de albañilería de adobe con aparejos a soga y tizón, tizón o inglés, o de los escasos ejemplos de piedra con aparejo ordinario o enripiado, en ambos casos dando como resultado espesores de muros de mayor dimensión (dentro de un rango de 40 a $80 \mathrm{~cm}$ ). Un segundo aspecto lo constituye la correlación que se da entre los patrones establecidos, el tipo de asentamiento y el sentido creciente en el que se presentan los valores de superficies construidas en favor de los casos asentados en áreas mayormente urbanizadas.

\subsubsection{Resultados obtenidos para MpG y comparación con MpPF}

El dendograma para MpG se construyó prácticamente en iguales condiciones que en MpPF. Se consideraron las primeras siete componentes principales del AFM para alcanzar una explicación del $62,80 \%$ de la variabilidad del modelo con la diferencia de que el incremento de casos de estudio conllevó la diferenciación de un cuarto patrón arquitectónico (Fig. 6). En esta representación se destaca una característica generalizada: distintas fases de una misma vivienda se agruparon entre sí en una primera instancia de aglomeración antes que con fases correspondientes a otras viviendas. Esto demuestra que, si bien la proximidad espacial entre viviendas como factor de aglomeración deja de ser tan fuerte como se presentó en los casos de MpPF (basta observar que acontece sólo de manera clara en los

\footnotetext{
5 Las quinchas (término de origen quechua) constituyen un caso específico de técnica de entramado, equivalente al waste and daub de la cultura constructiva anglosajona o a la técnica de clayonnage francés. Están conformadas por una estructura principal o armazón de maderas o ramas gruesas y un zarzo interno vertical. Este último, generalmente de cañas de Arundo donax o ramas de arbustos nativos que luego es embadurnado con barro en ambas caras.

6 Las cubiertas de torta, livianas o pesadas, son técnicas de entramados empleadas en los techos y se conforman por una sucesión de diversas capas de material vegetal en cuya capa superior se dispone una delgada capa de barro (la torta de barro propiamente dicha). La diferencia entre ambas radica en que la torta pesada emplea una gruesa capa de ramas, la enramada, como elemento sustentante de la torta de barro. En la torta liviana, esta estructura es reemplazada por una capa más lábil integrada por un cañizo de $A$. donax de escaso espesor y peso.

7 La incorporación de sistemas de acequias unificados con el fin de efectuar un uso más racional de los escasos recursos hídricos propició la aglomeración de las edificaciones en torno a poblados rurales menos dispersos. En algunos casos se observa la presencia de rutas provinciales y otras infraestructuras de servicio reforzando la estructuración de dichos poblados.
} 


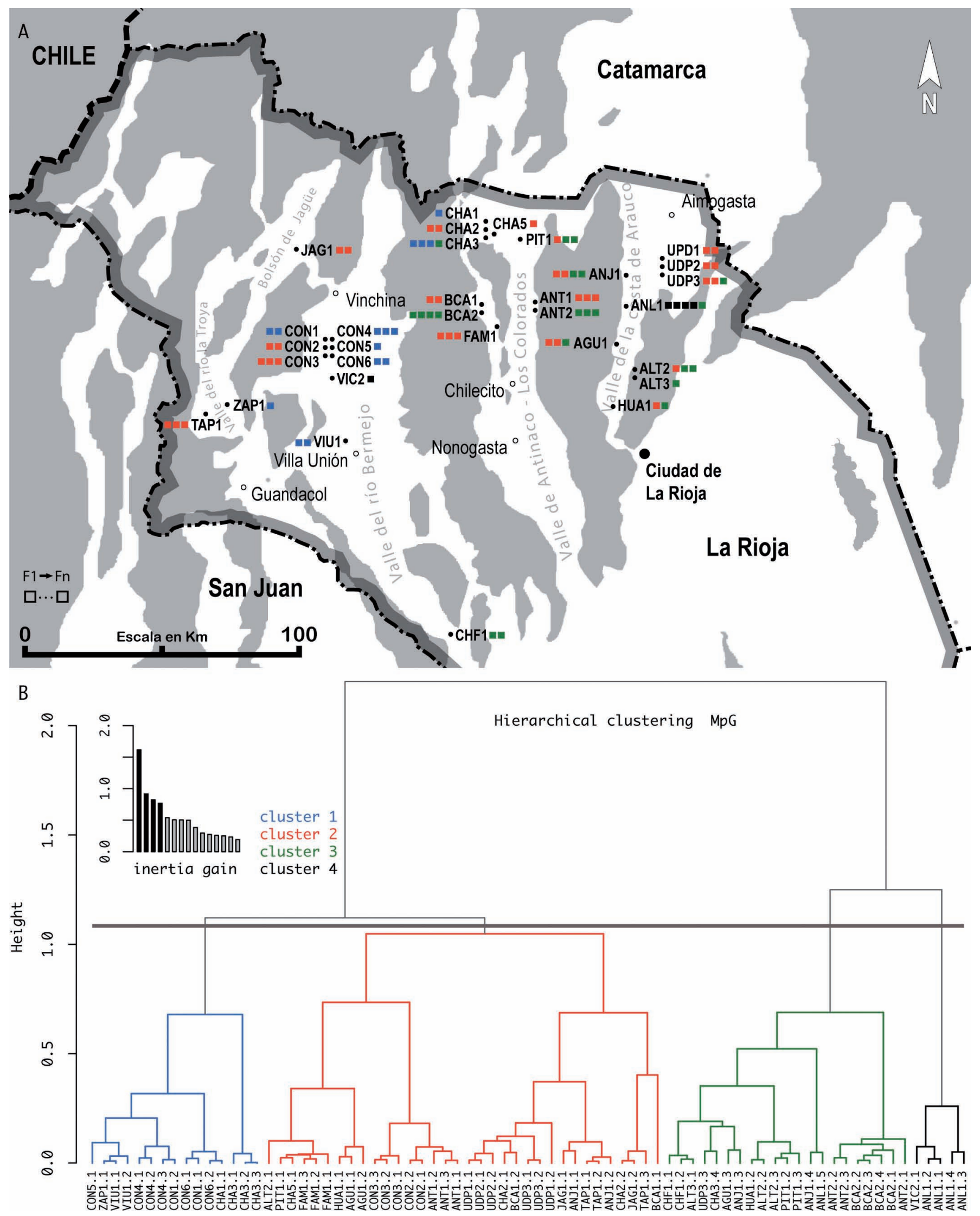

Figura 6. a). Distribución de los casos de estudios, los colores corresponden a los clusters establecidos para MpG; b) Cluster jerárquico de casos para MpG. Fuente: Elaboración propia. 
Factor map MpG

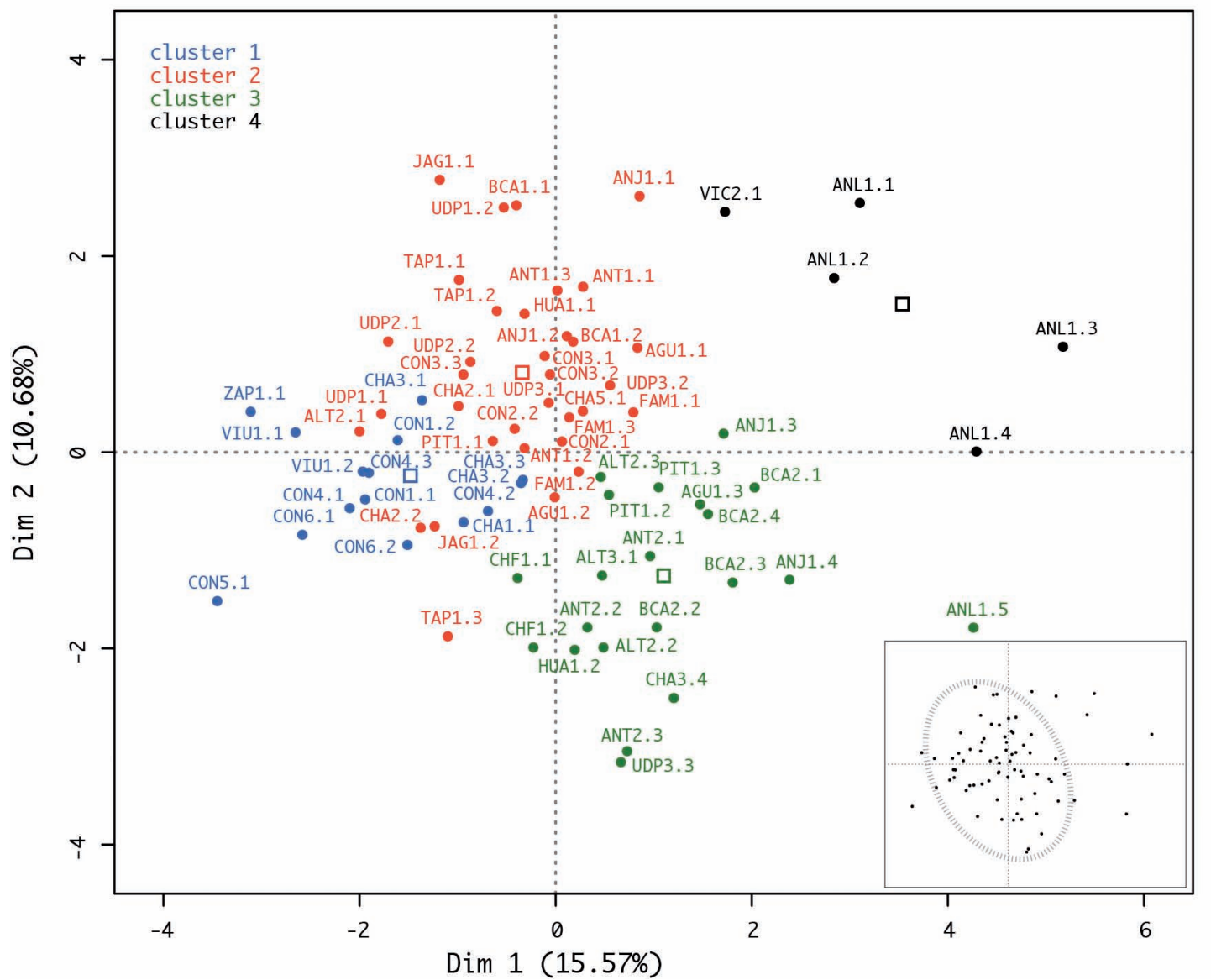

Figura 7. Representación de los clusters de la MpG en el plano inducido por los dos primeros componentes principales. Los cuadrados indican los baricentros de los respectivos conglomerados. En el extremo inferior derecho se indica el sector de mayor densidad de casos. Fuente: Elaboración propia.

grupos de casos correspondientes a las viviendas analizadas [CON1 - CON4 - CON5 - CON6] y [UPD1 - UPD2 - UPD3]), esta característica se traslada ahora a la relación entre fases constructivas de una misma vivienda.

Existen otras cuestiones más asociadas a la distribución de los casos aglomerados y su distribución en el territorio: en la Figura 6A se puede observar que la distribución del cluster naranja, el más numeroso, ocurre de manera homogénea entre los distintos valles, marcando una diferencia respecto de lo que se observa para el cluster equivalente de MpPF. Distinta es la situación de los clusters azul y verde que tienden a diferenciar sectores orientales y occidentales de distribución ${ }^{8}$.

La lectura combinada del gráfico de las variables activas y el gráfico que representa la contribución de cada grupo de variables (Fig. 4B) permite señalar los siguientes aspectos:

\footnotetext{
8 El cluster negro compromete sólo a fases constructivas de dos viviendas por lo que resulta insuficiente para proponer un patrón de distribución específico.
}

a) Existe una agrupación relativamente densa de casos (Fig. 7, elipse punteada) y otros casos que se alejan de este conglomerado. Aparentemente como producto del incremento de casos de estudio, la distribución de los mismos y de los baricentros de los cluster azul, naranja y verde de $\mathrm{MpG}$ resultan más aglomerados entre sí que en MpPF demostrando una distribución «de contagio». Por otro lado, una parte de los casos que se alejan del conglomerado de casos citado corresponden al cluster negro y presentan una clara distribución «de repulsión»?.

b) Los grupos de las variables tecnológicas y morfológicas se presentan igualmente correlacionados para ambos ejes del primer plano factorial (Fig. 4C, derecha). Por esta razón, a diferencia de lo observado

\footnotetext{
9 Categorías tomadas de la Taxonomía numérica y la Ecología, en donde se reconocen distintos tipos de distribución: «de contagio», cuando los casos tienden a aproximarse, «de repulsión» en sentido inverso y «uniforme» cuando ninguna de estas dos es preponderante.
} 
en MpPF, no es posible realizar una clara interpretación de los ejes factoriales en términos del tipo de variables que se les correlacionan. En este caso, la interpretación debió centrase atendiendo a las variables en forma individual: el conglomerado de casos relativamente denso se dispersan en función de las variables del grupo Superficies y de las variables tecnológicas TSOBREC y PENDTECHO (Fig. $4 \mathrm{C}$, izquierda). Las direcciones de estas variables conforman el espacio vectorial donde se produce la dispersión de los mismos.

c) Al igual que en MpPF, los casos CON5.1 y ANL1.1 seleccionados como control volvieron a presentarse como marginales respecto de la distribución general. Los casos de control adicionales para esta matriz, CHA3.4 y UDP3.3, también se presentaron como marginales y dentro de un mismo cluster. Entre los casos singulares se observan todos aquellos que integran el cluster 4, en particular el caso ANL1.5; incluso se puede señalar que el caso CON5.1 adquiere características de singularidad (Fig. 7).

\subsubsection{Patrones arquitectónicos en MpG: vigencias $y$ alteraciones hasta finales de la primera mitad del s. $X X$}

En la identificación de patrones arquitectónicos para $\mathrm{MpG}$, la vigencia de las características de los clusters azul y naranja respecto de MpPF se confirma si se toman en cuenta los casos que siguen integrándolos y su ubicación relativa al espacio vectorial que definen las variables (Fig. 7). Sin embargo, la mayor heterogeneidad de los rasgos que presentan los casos que lo integran explica la disposición de contagio que adquiere la distribución. Como se señalará más adelante, este aspecto es clave para comprender el proceso general de la variabilidad durante el recorte histórico considerado. Asimismo, la distribución territorial que refleja en particular el cluster naranja, ahora más amplia y homogénea para toda el área que lo observado para MpPF, podría interpretarse en esta línea argumentativa (Fig. 6A).

Distinta es la situación que presentan los clusters verde y negro. El primero de ellos es, fundamentalmente, un nuevo patrón arquitectónico. Éste es producto de la incorporación de fases constructivas de ampliación en la que se emplean en forma combinada tecnologías vernáculas e industrializadas como albañilerías que utilizan bloques de cemento y cubiertas de materiales prefabricados. El segundo es un patrón arquitectónico vinculado a asentamientos implicados en procesos de urbanización. Ello favoreció una mayor incorporación de rasgos tecnológico y morfológico urbanos que marcan una acentuada tendencia de diferenciación con el resto de los casos (por ejemplo, la inversión de la galería respecto del frente de la vivienda y la elevada altura de muros y superficies construidas).

\subsection{Articulación de variables: clusters constructivos homogéneos}

La primera articulación de variables para definir un cluster constructivo homogéneo se estableció a partir del dendograma de variables de McPF (Fig. 8, dendograma izquierdo) observándose la articulación de 12 rasgos (Fig. 9 y 10). Por lo que pudo apreciarse, la articulación de rasgos de variables que entraron en juego en este cluster constructivo homogéneo incluyeron aspectos de los tres grupos de variables considerados: tecnológicos, morfológicos y de localización.

En McG se presentó la posibilidad de establecer más de un cluster constructivo homogéneo; al menos dos en este caso (Fig. 8, dendograma central, y Fig. 9). Uno de ellos, el más numeroso, está formado por la articulación de 18 rasgos: doce de variables tecnológicas y seis de variables morfológicas. Algunos de estos rasgos son compartidos con el cluster constructivo homogéneo establecido para McPF (es el caso de los rasgos 1.A, 2.T, $4.40,8 . \mathrm{H}, 15.2$ y 25.P). El segundo cluster constructivo homogéneo está formado por siete rasgos. Este segundo conjunto se caracteriza por articular rasgos vinculados a la introducción de materiales y tecnologías de origen industrial.

De seis rasgos que son compartidos por McPF y $\mathrm{McG}$, cinco corresponden a variables tecnológicas (Fig. 9). En parte, la concurrencia de estos rasgos tiene un sentido lógico si tomamos en cuenta que los casos de estudios de McPF conforman un subconjunto dentro de McG. De todos modos, de las observaciones mencionadas se desprenden dos cuestiones que es preciso adelantar:

a) en relación a los rasgos de variables compartidos: su persistencia, luego de la incorporación significativa de casos para $\mathrm{McG}$, sugiere que la relación establecida entre las variables resulta robusta (en términos estadísticos), suficiente como para mantenerse vinculadas frente a la aparición de nuevos casos (nuevas viviendas o fases constructivas) y rasgos (introducción de nuevos materiales y/o técnicas constructivas). 


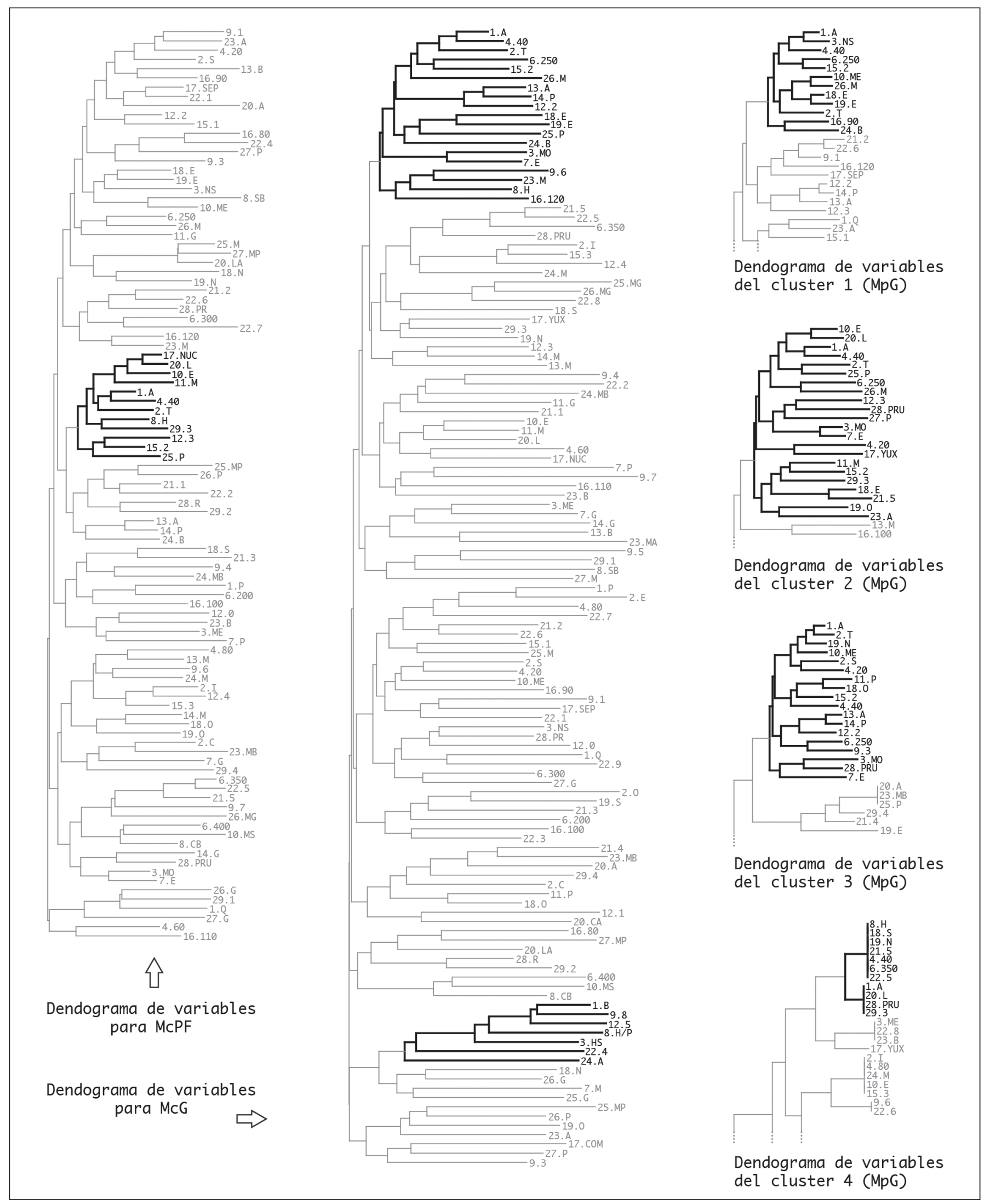

Figura 8. Clusters constructivos homogéneos: dendogramas de las variables para McPF, McG y para los distintos patrones arquitectónicos obtenidos en MpG. Fuente: Elaboración propia. 


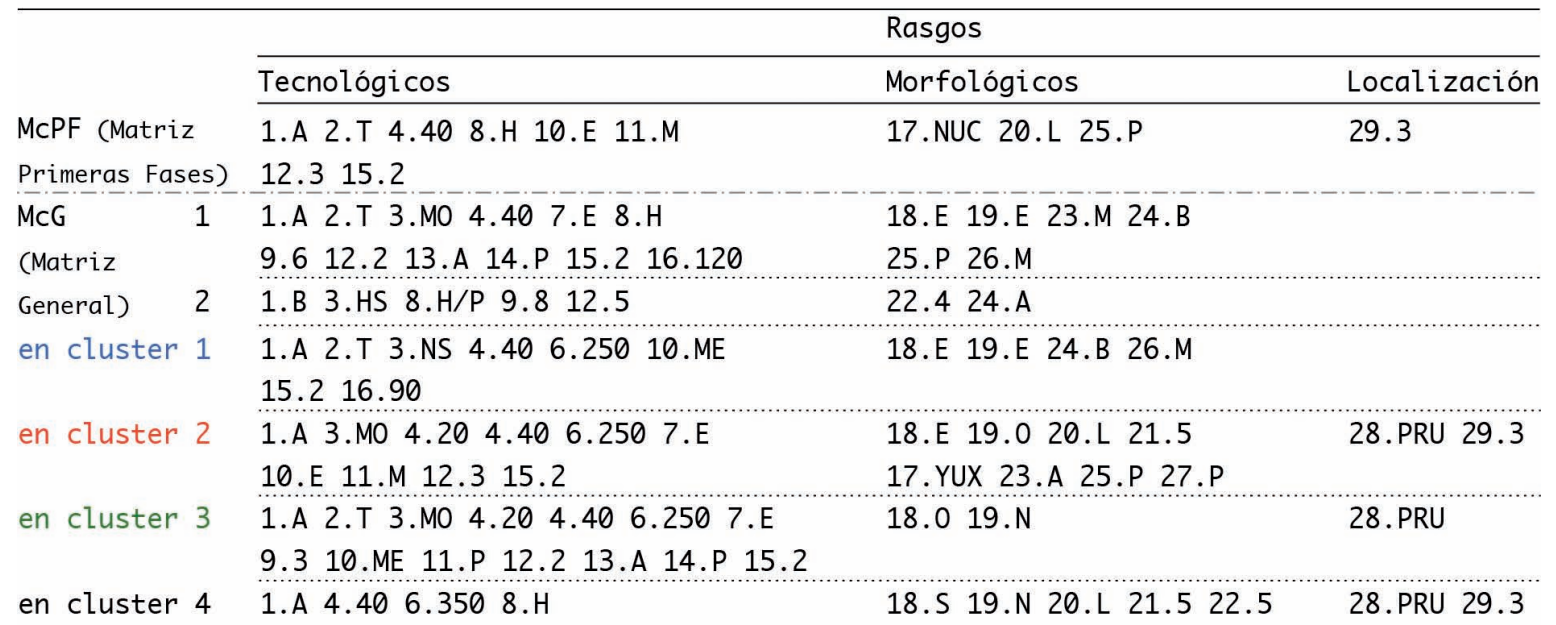

Figura 9. Clusters constructivos homogéneos: rasgos estructurados para cada una de las matrices y ordenados según tipos de variables. Fuente: Elaboración propia.

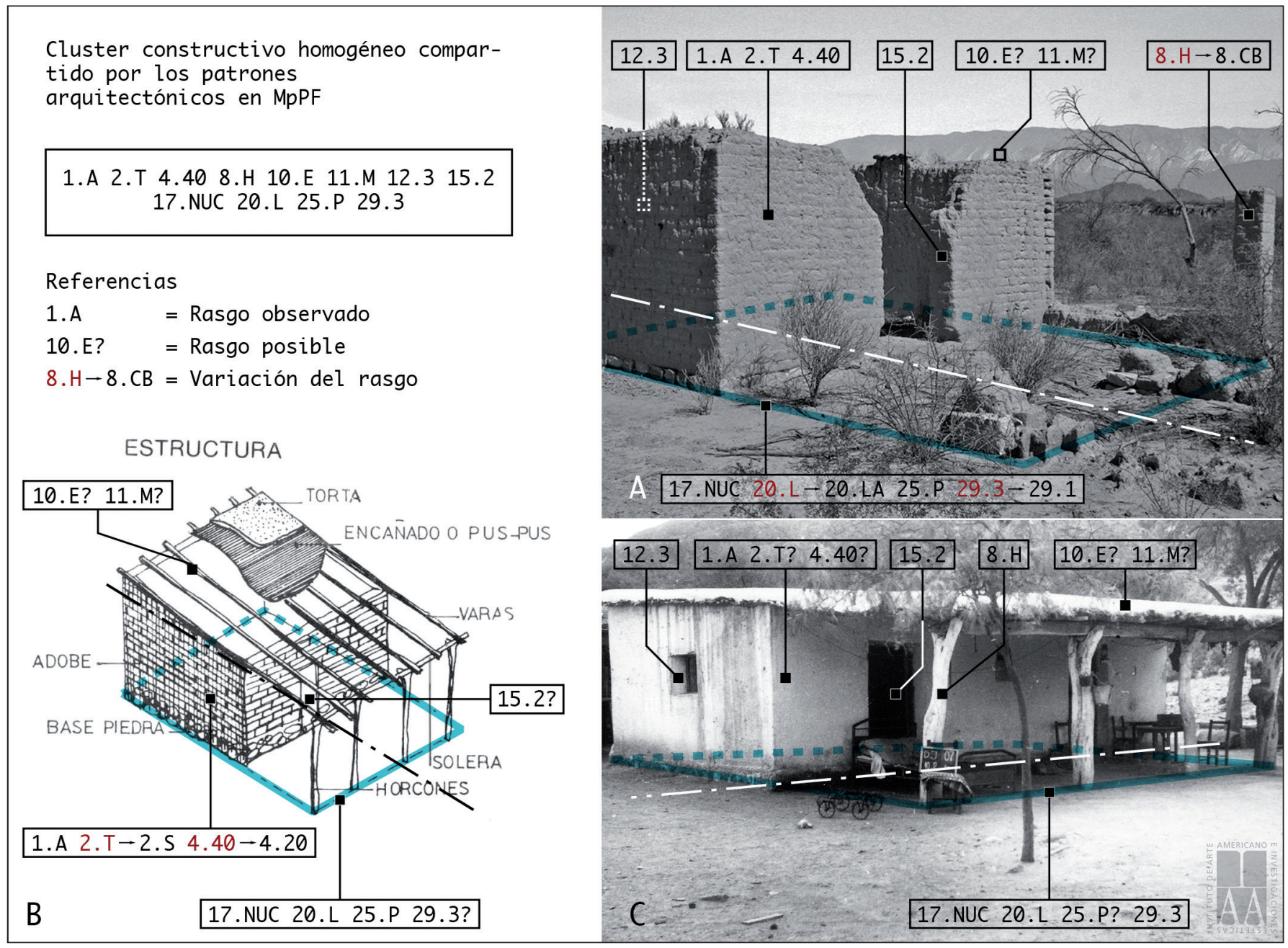

Figura 10. Aplicación del Cluster constructivo homogéneo y posibles variaciones utilizando el cluster obtenido para MpPF. a) Caso CHA1.1 (Fuente: Colección personal del autor); b) Representación de vivienda típica para la región de valles riojanos (Fuente: Instituto de Investigaciones de la Vivienda 1972:121); c) Vivienda habitada, ca. 1960 (Fuente: Fototeca del Instituto de Arte Americano e Investigaciones Estéticas, autor anónimo). 
b) a partir de lo observado en la McG: es posible identificar simultáneamente más de un cluster constructivo homogéneo dentro de una misma matriz. Pero esto está sujeto a que la relación entre los rasgos que integran cada cluster constructivo homogéneo no se presente compartida. Es decir, si uno o varios rasgos pueden formar parte de dos o más clusters constructivo homogéneos, estos rasgos sólo se asociarán al cluster que se estructuran de manera más robusta. Ante esta situación, una alternativa que permite la metodología para lograr identificarlos separadamente, es la construcción de matrices parciales. Por ejemplo, se pueden conformar matrices parciales a partir de separar los datos asociados a los casos que forman cada patrón arquitectónico.

Para ejemplificar lo mencionado en el segundo punto, se realizó el clustering sobre matrices parciales de $\mathrm{McG}$ obteniéndose clusters constructivos homogéneos para cada uno de los patrones arquitectónicos (Fig. 8, dendogramas derechos ${ }^{10}$. Los resultados confirman lo que se señaló anteriormente: la existencia de clusters constructivos homogéneos distintos formados por algunos rasgos de variables que se comparten. En general, entre los clusters constructivos homogéneos obtenidos para cada uno de los patrones arquitectónicos de $\mathrm{McG}$, los principales rasgos compartidos que se observaron fueron los tecnológicos, lo que nos conduce a sugerir que, efectivamente como sugiere Sánchez Zufiaurre (2007), constituyen los rasgos más estables frente a la variabilidad general.

\section{Discusión}

\subsection{El carácter de los estudios tipológicos para el área}

La estudios existente referidos a la arquitectura rural de la provincia de La Rioja no sólo han resultado escasos (Aparicio 1937; Cáceres, 1946; Armellini, Cóppola, Iglesias y Rosso 1970; Canepuccia, Castro, Ocvirk y Ostroposky 1976), sino incluso insuficientes para comprender de manera más precisa la incidencia de los procesos históricos en la materialización de las viviendas populares. Asimismo, estos antecedentes no se han centrado en esclarecer los motivos que condicionaron el surgimiento

\footnotetext{
10 Sin embargo, para las matrices parciales de McPF este procedimiento no permitió obtener resultados con un grado de certidumbre suficiente para ser propuestos debido al escaso número de casos que integraban cada uno de los clusters arquitectónicos.
}

o abandono de los patrones arquitectónicos observados; en general, se han remitido a señalar simples razones de disponibilidad de recursos, reproducción de soluciones existentes e introducción de conocimientos técnicos por parte de nuevos pobladores. Por otra parte, los estudios de Aparicio (1937) y Armellini, Cóppola, Iglesias y Rosso (1970) fueron los únicos realizaron un intento por diferenciar algunos tipos arquitectónicos para el ámbito rural, siendo sólo los segundos quienes aventuraron una explicación de las razones (principalmente socioeconómicas en este caso) vinculadas a tal proceso.

El trabajo de Armellini, Cóppola, Iglesias y Rosso (1970) constituye un referente importante para señalar, entre otras cosas, aquellos problemas o limitaciones metodológicas recurrentes que se observan en los estudios tipológicos para el área y, por extensión, para aquellos estudios que se sirven del concepto de «tipo edilicio». Este trabajo es el antecedente más cercano, contiene numerosos casos de observación considerados y presenta un análisis que comienza a tomar en cuenta la complejidad involucrada en la producción de la arquitectura doméstica. Sin embargo, al recurrir al concepto de «tipo edilicio» para establecer la estructura clasificatoria propuesta se observa que los autores no se involucraron en un análisis tipológico más profundo al no considerar los procesos de variabilidad sincrónica entre los casos observados y al no tomar en cuenta transformaciones socioeconómicas y tecnológicas que se estaban desarrollando contemporáneamente en el área de estudio. Desde el punto de vista teórico esta profundización no se produce debido a que el concepto de «tipo de edificación» no requiere especificar la red de relaciones que en el «patrón arquitectónico» está presente por definición. De este modo, una contextualización más profunda de los tipos edilicios identificados queda librada al interés del investigador y no por ser parte del marco teórico escogido y la metodología de análisis implementada.

Para ejemplificar esta problemática observada podemos remitirnos a las categorías establecidas por estos autores para el área rural en particular: los tipos edilicios identificados en el estudio citado fueron denominados recurriendo en varios casos a términos nomotéticos que pusieron en evidencia el problema de reiterar modelos explicativos preestablecidos y el uso de denominaciones ambiguas para el establecimiento de su clasificación. Nos referimos a tipos edilicios designados bajo los apelativos de «Ranchos», caracterizados por resoluciones precarias y para los que sugieren un origen prehispánico 


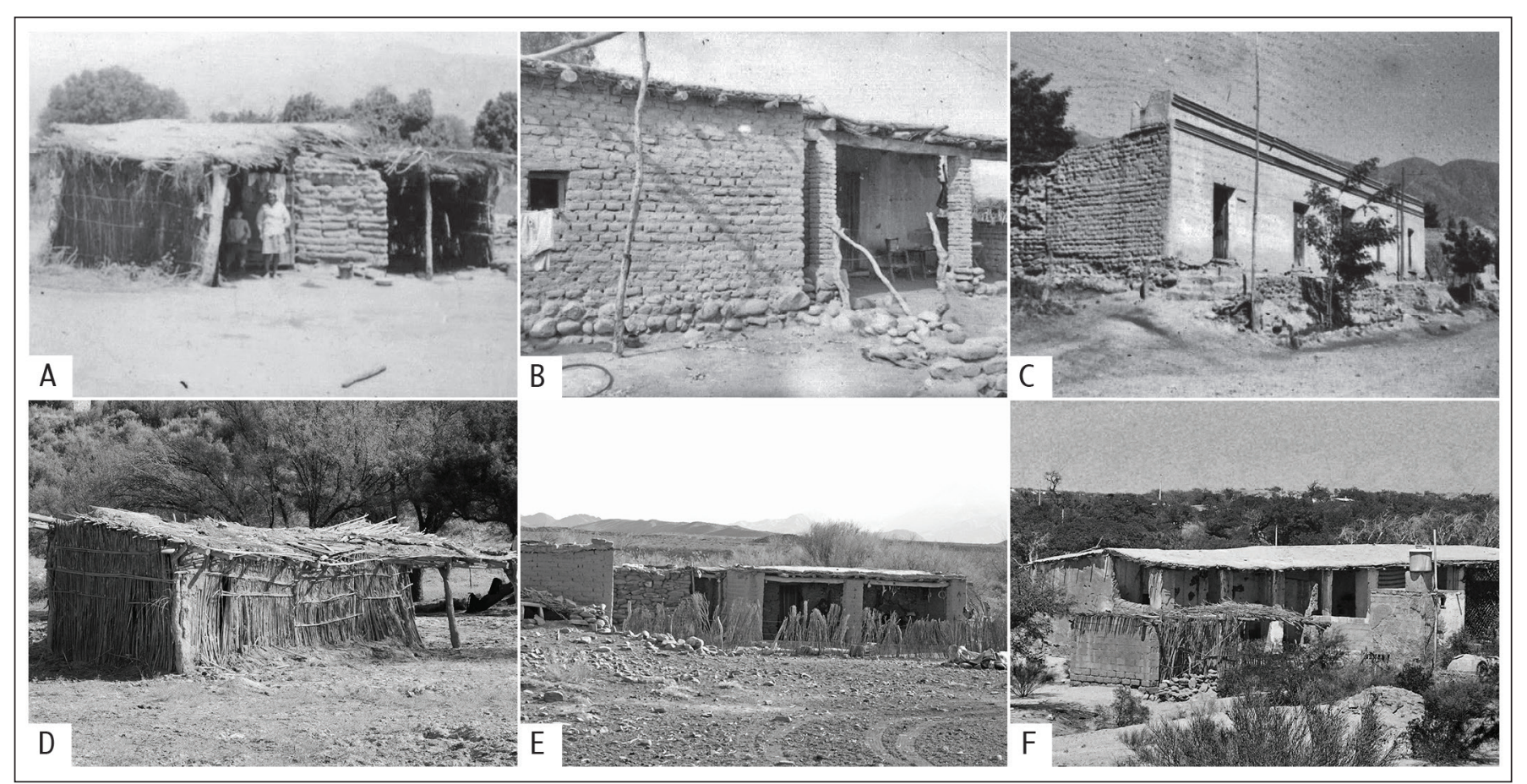

Figura 11. Viviendas rurales riojanas: a, b y c) ejemplos registrados e indicados por Armellini, Cóppola, Iglesias y Rosso (1970) para «Rancho», «Vivienda de transición» y «Casa Criolla» respectivamente; d, e y f) ejemplos para los tres principales patrones de MpG: CON5 (patrón 1), TAP1 (patrón 2) y UDP3 (patrón 3), respectivamente. Fuente: Colección personal del autor.

en sus formas y soluciones ${ }^{11}$; «Viviendas de transición», las cuales resultarían de procesos en los que se van incorporando tradiciones constructivas de las poblaciones colonizadoras a los patrones arquitectónicos propiamente desarrollados por la vivienda prehispánica ${ }^{12}$; y finalmente, «Viviendas criollas», edificaciones que

\footnotetext{
11 Esta asociación también es sugerida por Chiozza y Aparicio (1961: 523531). Sin introducirse en esta discusión en particular, las viviendas en cuestión fueron producto de las estrategias desarrolladas por un segmento de la población local frente a duras condiciones sociales e históricas que debieron afrontar ante unas situaciones de relativo aislamiento social y espacial, frente a un medioambiente altamente hostil y en condiciones de pobreza estructural. Este patrón de viviendas casi no fue registrado en el estudio fundamentalmente por la lábil y efímera conformación con las que fueron resueltas y a su escasa perdurabilidad frente a los agentes ambientales. Por otra parte, el término «rancho» ha sido empleado a lo largo de todo el territorio argentino, muy diverso en términos geográficos y culturales, para señalar manifestaciones constructivas extremamente disímiles (Instituto de Investigaciones de la Vivienda 1972)

12 Los autores emplean el término «Viviendas de transición» para resolver la denominación del grupo más diversamente representado tanto en su registro como en el que se presenta en este estudio. Sin embargo, el aspecto más importante que se desprende de esta designación es una aparente interpretación de los procesos históricos en términos evolutivos (casi lineal) como resultado del contacto con tradiciones constructivas occidentales; los autores parten de la tipología de «Rancho» (aparentemente en términos arquitectónicos menos evolucionadas) para alcanzar la tipología de «Vivienda criolla» (más evolucionadas). Es evidente que la simplificación en el análisis de los procesos históricos estuvo cargada de un importante contenido de prejuicios y de evaluaciones $a$ priori.
}

comienzan a incorporar rasgos urbanos como, por ejemplo, la inversión de la galería frontal, el desarrollo de fachadas de gran altura hacia la calle, muchas de las cuales se localizan, sin embargo, en ámbitos principalmente rurales o en la periferia de los pocos espacios urbanos que existen en los valles (Fig. 11). Sosteniendo lo que sugiere Sánchez Zufiaurre (2007), recurrir a este tipo de reducciones en etapas iniciales del estudio supone un fuerte inconveniente dada la complejidad a que está sujeta la producción de la arquitectura, conduciendo con ello a evaluaciones incompletas y sesgadas.

\subsection{Patrones arquitectónicos y variabilidad}

Es en función del mayor o menor grado de relación que se establece entre los casos de estudio (es decir, su similitud o disimilitud) lo que permite determinar clusters $\mathrm{y}$, a partir de las características del análisis que se realice sobre los resultados, arribar a tipos o patrones. Al mismo tiempo, si se atiende a la variabilidad interna y entre agrupamientos, y se identifican aquellas variables más importantes que son responsables de tal proceso de «variabilidad sincrónica», también es posible reconocer con qué grado de regularidad son definidas las clases tipológicas establecidas. Para ello, el concepto de variabilidad se interpretó en este estudio como el espacio 
pluridimensional definido por un conjunto específico de variables donde se produce la distribución y entran en relación los casos de estudio definidos. En general, los estudios tipológicos desde la disciplina arquitectónica han indagado escasamente en los procesos de variabilidad sincrónica, y en particular en la interpretación de las causas que van marcando las diferencias o disimilitudes entre casos. Esto se debe a que la mayor atención ha estado puesta en la identificación de aquellos rasgos que permiten establecer las similitudes. Por otra parte, la literatura se ha focalizado principalmente en analizar los procesos de «variabilidad diacrónica» al dar mayor importancia a las variaciones producidas que permitieron explicar, por ejemplo, el pasaje de un tipo edilicio a otro (Diez 1996: 31) o el tránsito desde el prototipo hasta el estereotipo (Martín 1984: 173-175), y que forman parte de lo que se ha denominado «proceso tipológico» (Caniggia y Maffei 1995: 31-32). Sin embargo, en la definición del patrón arquitectónico, y a diferencia del de tipo edilicio, ambos conceptos de variabilidad constituyen rasgos fundamentales que permitirían explicar la capacidad de reproducción y adaptación del patrón arquitectónico al contexto de aplicación.

Para el presente estudio, el espacio de variabilidad fue representado en MpPF por los mismos ejes de representación (recordemos la correlación de los grupos de variables tecnológicas con el primer eje y de las variables morfológicas con el segundo). Por su parte, en MpG se encontró definido por un conjunto específico de variables que estableció la dirección principal sobre el que se desarrolló la diferenciación de la masa crítica de los casos de estudio y la distribución por contagio de los tres patrones arquitectónicos más importantes. En este segundo caso, observando el sector del eje menor de la elipse de la Figura 5, se aprecia que el resto de las variables representadas, complementariamente, terminan de definir este espacio de variabilidad al dejar en evidencia la dirección más conservadora de la misma. Sólo cuando los rasgos de la mayor parte de las variables involucradas en dicha dirección son suficientemente divergentes se produce la diferenciación de un nuevo patrón arquitectónico (cluster negro). Este suceso en la distribución general de MpG puede interpretarse en los siguientes términos: si bien existe una recurrencia de aspectos que son los que conducen a la constitución de los patrones arquitectónicos, también es posible sugerir que la «permutabilidad de rasgos» entre casos de distintos clusters es un hecho importante que está presente y que provoca la distribución por contagio entre los tres principales patrones arquitectónicos obtenidos; de no existir tal permutabilidad, el gráfico debería haber experimentado una distribución de repulsión entre los distintos patrones arquitectónicos establecidos (semejante a la distribución observada para $\mathrm{MpPF}$, en la cual la distribución por contagio es menos acentuada). Argumentación que puede ser reforzada si se toma en cuenta otros dos aspectos: la forma de distribución por repulsión del cuarto patrón arquitectónico (cluster negro) de MpG y si se comparan los rasgos que integran cada cluster constructivo homogéneo de los patrones arquitectónicos obtenidos.

Los casos de estudio que integran la masa crítica en $\mathrm{MpG}$, y dan la forma característica de distribución por contagio, se corresponden con la categoría «Vivienda de transición» de la clasificación propuesta por Armellini, Cóppola, Iglesias y Rosso (1970). Esta circunstancia pone en evidencia que tal tipo edilicio sería el patrón arquitectónico más extendido en el área rural para el período considerado. Sin embargo, según las observaciones de estos autores (Armellini, Cóppola, Iglesias y Rosso 1970: 10), el citado patrón habría ido perdiendo vigencia progresivamente desde inicios del $\mathrm{s}$. XX hasta ser finalmente abandonado a mitad del mismo.

Otro aspecto a señalar en la distribución de $\mathrm{MpG}$ es aquel en el que se aglomeraron los casos que introducen materiales y tecnologías industrializadas como parte de las resoluciones técnicas incorporadas (cluster verde). Si bien estas incorporaciones son parciales y no plantean modificaciones sustanciales de las estrategias morfológicas y de crecimiento de las viviendas diferentes a las desarrolladas para el período, son suficientes para plantear una tendencia en la diferenciación entre clusters desde el punto de vista tecnológico. Se observaron cambios muy graduales desde la simple incorporación de bloques de cemento en la construcción de algunos muros donde antes se recurría al uso de albañilerías de adobe o piedra, hasta la incorporación de todo un sistema constructivo (casos CHF1.2, CHA3.4, UDP3.3 y ANJ1.4). Este proceso, que se inicia recién en el transcurso de las décadas de los años 50 y 60 del s. XX en el ámbito rural, no parece haber sido identificado como algo significativo por estos autores. Por otra parte, CON5.1 (Fig. 11D) es el único caso de estudio prospectado en esta investigación que se corresponde con el tipo edilicio «Rancho» de la misma clasificación tipológica señalada. Es posible que esta circunstancia responda al fácil deterioro estructural de estas unidades domésticas debido a lo lábil y efímero de su conformación frente a los agentes ambientales, pero también podemos sostener, recurriendo al concepto 
de «proceso tipológico» de Caniggia y Maffei (1995: 31-32), que tal caso de estudio no es más que la expresión remanente de un patrón arquitectónico que para el período considerado había comenzado a caer en desuso. Este tipo edilicio prácticamente no fue observado en el estudio y por ello el caso se incorporó de manera premeditada en el estudio como elemento de control con el fin de contar con otro instrumento de evaluación del proceso de variabilidad.

\subsection{Las condiciones del contexto histórico y socioeconómico}

Desde el período colonial (fines del s. XVI a inicio del s. XIX) y hasta mediados del republicano (s. XIX a la actualidad), la provincia de La Rioja mantuvo un creciente intercambio comercial extrarregional con el Centro y Norte de Chile. Este intercambio específico, que inicia su fase de decadencia entre fines del s. XIX y las primeras décadas del siguiente, resultaba el principal motor de la actividad económica de las poblaciones rurales riojanas (Olivera 2001a; Fuente 2007). Hacia el final de este ciclo, y con la intención de contrarrestar la progresiva situación de desvinculación comercial que se estaba produciendo, se intentó reforzar el endeble comercio que se mantenía con el resto de las provincias argentinas centrándose fundamentalmente en las actividades extractiva forestal, minera, vitivinícola y en el estímulo de la producción olivícola, pero sin alcanzar mayores ventajas inmediatas. De todas formas, tanto antes como durante el proceso de reorientación comercial, el arreado de ganado vacuno y el transporte de otras mercancías (fundamentalmente vino y aguardiente) sustentaban una práctica trashumante muy extendida que propició, además del establecimiento de los lazos comerciales (y que logró, como señala Olivera (2000), una favorable articulación con el servicio ferroviario establecido hacia fines del s. XIX), un intercambio de informaciones y conocimientos permanente entre el rosario de poblaciones asentadas en las diversas rutas establecidas.

En este contexto, la extrema escasez hídrica fue uno de los factores de mayor complejidad que debió enfrentar la población rural. Las acciones de los distintos gobiernos provinciales no lograban encontrar solución para revertir tal situación y propiciar el aumento de la productividad agrícola. Recién en la década de los años treinta del s. XX se da inicio a diversas obras de embalse, explotación de acuíferos y mejoras en las redes de acequias que posibilitaron el incremento en la reserva del recurso hídrico y un menor desperdicio en su distribución. Es por ello que, hasta pasado el primer cuarto del s. XX, no existieron posibilidades de expandir la frontera agropecuaria en ninguno de los poblados de la provincia (Cresta y Suárez 1970; Olivera 2000).

A pesar de las circunstancias referidas es evidente que entre la segunda mitad del s. XIX y hasta las primeras décadas del siglo siguiente existieron ciertas condiciones, aunque exiguas, para el establecimiento de nuevas unidades domésticas y una modesta prosperidad económica de la población rural. La limitada expansión económica, propiciada por el valor agregado de las renovadas actividades productivas, permitió sostener este proceso de establecimiento de la población hasta las primeras décadas del s. XX (Barros, Thierry, Huergo y Alsina 1894; Sánchez 1928). Este bienestar acotado permite explicar, en cierta medida, la antigüedad de las viviendas analizadas en este estudio y las ampliaciones que se observaron en las mismas como por aquellas informadas en investigaciones previas (Armellini, Cóppola, Iglesias y Rosso 1970: 35).

Si bien durante las décadas de los años 20 y 30 del s. XX acontecieron algunos importantes avances técnicos y de infraestructura para mejorar la producción agropecuaria en la región ${ }^{13}$ (Díaz 1974), en simultaneo se desencadenaron factores externos (fundamentalmente la demanda de mano de obra debido a procesos de intensa industrialización de las urbes) que propiciaron un repentino e intenso proceso de emigración de la población rural. A este panorama se sumaron factores internos también importantes: los jefes de familia de la población comprometida eran minifundistas (estancieros o labradores), trabajadores asalariados, obreros, pastores o peones que no poseían la propiedad de la tierra (Armellini, Cóppola, Iglesias y Rosso 1970: 13). La irregularidad en los títulos de propiedad de las tierras rurales (o títulos imperfectos, como se los denominaba) se mantuvo finales del s. XX, con lo cual a muchos productores rurales se les hizo imposible acceder a préstamos bancarios para realizar inversiones, mejorar el equipamiento para las tareas rurales o comprar semillas (Olivera 2001b). Debido a ello, se vieron impedidos de aprovechar los programas oficiales de estímulos para incrementar su capacidad productiva. En paralelo, la venta de cuotas de

\footnotetext{
Numerosas obras de infraestructura hídrica posibilitaron incrementar el volumen de agua de reserva en numerosos poblados y, con ello, disponer de un mayor número de «cuotas de agua» (horas de riego). Esta circunstancia posibilitó el inicio de los procesos de explotación agrícola extensiva y se comienzan efectuar algunos casos de explotación agrícola intensiva como sucede con el proceso de «Olivitización» en las áreas olivícolas (Olivera 2001b).
} 
agua entre particulares constituyó una práctica común a la cual recurrían los pobladores para resolver transitoriamente sus problemas económicos, viendo reducida de tal forma su capacidad productiva. A su vez, el otorgamiento de nuevas cuotas de agua no se efectuó sobre una base de distribución equitativa. Olivera (2001b) señala que el «amiguismo», el «clientelismo político» (y podría agregarse que la posición dominante en el mercado de determinados actores como propietarios latifundistas, familias tradicionales o representantes políticos de la administración provincial) propiciaron o efectuaron, durante todo el período, un proceso de concentración de cuotas de agua en pocos regantes, aspecto que hoy en día da cuenta del paisaje cultural como se puede apreciar (Rolón y Rotondaro 2011).

Este conjunto de circunstancias mencionadas desencadenó, hacia finales del período considerado, un proceso en el cual gran parte de la población rural de los valles encontró seriamente dificultada la posibilidad de afianzarse económicamente y, como ya se señaló, se encontró obligada a migrar al ámbito urbano en busca de mejores expectativas de desarrollo. Este proceso señalaría el tiempo en que acontecieron los abandonos registrados de gran parte de las viviendas analizadas en el estudio.

\subsection{El horizonte tecnológico en la arquitectura rural riojana}

La tierra como material de construcción constituyó el principal recurso tecnológico elegido para resolver la materialidad de las viviendas rurales populares en los valles riojanos. Si bien es algo que se observó durante las prospecciones, varios de los rasgos incluidos en los clusters constructivos homogéneos obtenidos en todos los casos son concluyentes en este aspecto. La primera impresión nos aventuraría a proponer que la disponibilidad de este material constructivo de origen natural explicaría tal elección. Varios de los autores citados a lo largo de este estudio se han inclinado por esta posibilidad (Aparicio 1937; Chiozza y Aparicio 1961; Armellini, Cóppola, Iglesias y Rosso 1970). Sin restar cierto grado de veracidad a esta apreciación, no resulta suficiente para explicar por qué otros materiales naturales, como la piedra, también abundantes en la región, no fueron aprovechados con igual o mayor frecuencia que la tierra. Tampoco logra aclararse este interrogante si verificamos que el empleo de la albañilería de ladrillos no se utilizó en la región como sí aconteció en gran parte de la región de la pampa húmeda o incluso en Córdoba, provincia con la que mantuvo un persistente vínculo comercial y cultural; de hecho, la vinculación económica y espacial existente con esta provincia hubiese posibilitado el comercio de este producto y la sostenibilidad de la industria ladrillera con la introducción del ferrocarril.

Aquí es donde entra en juego la consideración de los factores de orden cultural y las estrategias de producción y reproducción técnica en el territorio. Viñuales (1991) señalaba que la arquitectura con tierra en la región andina ha contado con una sólida presencia desde períodos prehispánicos, en la que si bien se han ido descartando ciertas técnicas específicas (como el caso de la técnica del tapial), no por ello se vieron interrumpidos los procesos que permitieron la continuidad de estos conocimientos hasta avanzado el Período Republicano. Incluso las tecnologías de construcción con tierra continúan vigentes en el espacio social del Noroeste argentino (Pastor 2000; Tomasi y Rivet 2011).

La introducción de materiales industrializados en el último tiempo por iniciativa de los pobladores locales es otro de los tópicos presentes. Este proceso no significó por ello el abandono de sus tradiciones vernáculas de construcción pero, en conjunción con otros factores pre$\operatorname{sentes}^{14}$, sí afectaron negativamente el volumen de producción de la construcción con tierra en el ámbito rural.

Es posible discutir si la incorporación de materiales industrializados en la actualidad supone cambios sustanciales en las tradiciones vernáculas de construcción. Pero esto no significa que las mismas no hayan estado en transformación permanente en el pasado por la interrupción o la incorporación de otras técnicas y materiales. Estos aspectos permiten suponer que, si bien el horizonte tecnológico de la vivienda rural estuvo asociado a patrones tecnológicos que han hecho uso de la arquitectura con tierra, este horizonte difícilmente se ha mantenido estático. Ya de por sí, la identificación de clusters constructivos homogéneos relativamente diferentes para cada patrón arquitectónico presupone variaciones. Por citar un ejemplo del ámbito local, Armellini, Cóppola, Iglesias y Rosso (1970: 117) señalan que el empleo de cubiertas de tortas livianas responde a tradiciones constructivas procedentes

\footnotetext{
14 En los sectores próximos a las poblaciones urbanas, acontecieron prácticas discursivas y políticas que hegemónicamente menospreciaron determinadas experiencias culturales, la asociación de este tipo de manifestación arquitectónica con condiciones de insalubridad y precariedad, la discontinuidad en la transmisión de los saberes constructivos a causa de procesos migratorios mencionados y/o la falta de valoración de sus diversas potencialidades originaron malas prácticas en la intervención sobre construcciones existentes, su destrucción y, más controversial aún, el abandono progresivo del bagaje cultural en torno a esta tecnología.
} 
de la región cuyana, en tanto que las cubiertas de tortas pesadas tienen mayor afinidad con los patrones tecnológi$\cos$ de la vivienda rural santiagueña ${ }^{15}$. En igual sentido, es posible también reconocer que los dinámicos circuitos comerciales regionales y extrarregionales y la trashumancia característica de estos pobladores rurales (Olivera 2001a; Fuente 2007) propiciaron los procesos de reproducción técnica y cultural. Como señala Navarro (2006), estos procesos son característicos de la arquitectura popular y posiblemente favorecieron la homogeneización y la extensión de estas estrategias, aún en lo vasto de este territorio.

\section{Consideraciones finales}

En general, se ha considerado que la cultura popular tiende a ser conservadora en la proposición y resolución técnico-formal de sus espacios construidos. En este sentido, es evidente la existencia de un patrón de racionalidad común durante el período y para el extenso espacio geográfico considerado en este estudio. La existencia de pocos patrones arquitectónicos, la tendencia a una distribución por contagio de los casos de estudio (en parte por un proceso de permutabilidad de rasgos) y la presencia de un horizonte tecnológico consolidado en torno a la arquitectura en tierra son algunos de los aspectos que corroboran este punto de vista. Sin embargo, los conceptos teóricos aplicados y la metodología de análisis implementada para identificar patrones arquitectónicos y sus respectivas estructuras internas resultaron claves para dejar en evidencia y caracterizar la variabilidad sincrónica que se observó en la construcción de las viviendas rurales analizadas; variabilidad que responde al contexto específico de aplicación y a la dinámica de los procesos sistémicos en los que se desarrolla el hábitat popular.

Como se mostró a lo largo del trabajo, un patrón arquitectónico puede ser definido por dos características principales: su estructura interna, que permite caracterizar sus aspectos más estables y diferenciarlo respecto de otros patrones, y su margen de adaptación. Es por ello que la metodología presentada fue estructurada con el fin de poder determinar no sólo los patrones arquitectónicos, sino sus respectivas estructuras internas y, al mismo tiempo, el espacio de variabilidad o margen de adaptación del patrón al caso específico de aplicación. Por lo tanto, la variabilidad sincrónica y el cluster constructivo homogéneo son

\footnotetext{
15 La primera corresponde a una región argentina que limita al Sudoeste con la provincia de La Rioja, conformada por las provincias de Mendoza, San Juan y San Luis. La segunda a un gentilicio de la provincia de Santiago del Estero, hacia el sector Noreste.
}

conceptos complementarios que permiten caracterizar un patrón arquitectónico y definir su alcance de aplicación.

Para finalizar es oportuno señalar dos aspectos sobre los cuales se abre la posibilidad de profundizar en nuevos estudios a partir de los resultados obtenidos ${ }^{16}$ :

El primero de ellos es en relación a la metodología: en este estudio no se consideraron variables de orden social y cultural para ser aplicadas en el análisis multivariante. Esto se debe a la instancia exploratoria en la que se encontraba el proceso metodológico propuesto pero, sin duda, constituye una línea de trabajo sobre la cual progresar. La inclusión de este tipo de variables permitirá, en tanto sea posible lograr una codificación adecuada de los rasgos para efectuar el análisis, mejorar las interpretaciones sobre los procesos de variabilidad, ampliar la caracterización de los patrones arquitectónicos e indicar la posible existencia de procesos de complejidad social.

El segundo aspecto está en relación al tema abordado: la investigación se ha concentrado en el período previo a dos acontecimientos de profunda transformación en el territorio rural: el inicio de las políticas de intervención estatal en el ámbito de la vivienda de interés social en La Rioja, y con ello la introducción de un actor institucional y dominante en la programación y diseño sistematizado de la vivienda, y el desencadenamiento de un desarrollo agroindustrial más incipiente. Ambas circunstancias son factores principales y nuevos que podrían estar implicados en la transformación del patrón de racionalidad y del horizonte tecnológico del período previo al actual, con un impacto sustancial en la producción material y cultural del espacio doméstico. Analizar y comparar ambos períodos desde estos puntos de vista son líneas de investigación que podrían aclarar aspectos importantes de los procesos tipológicos de la vivienda doméstica en los dos últimos siglos.

\section{Agradecimientos}

El encuadre teórico y metodológico que se exploró para llevar a cabo esta investigación, vinculada al campo disciplinar de la Arqueología de la Arquitectura, fue posible gracias al fortuito vínculo establecido con Agustín Azkarate desde 2009. Quiero señalar mi más sincero agradecimiento a su persona por su total apoyo, compartiendo sus conocimientos e incluso, convencido de los objetivos promisorios de la investigación, promover

\footnotetext{
16 Aspectos que surgen del intercambio con los evaluadores.
} 
mediante la gestión y el financiamiento necesarios mi estadía en su grupo de investigación. Asimismo, quiero agradecer la importante colaboración de Marcelo Cardillo quién dedicó desinteresadamente parte de su tiempo para introducirme en el uso e interpretación de las técnicas de análisis multivariante en arqueología. No puedo dejar de mencionar las aportaciones de Leandro Sánchez Zufiaurre y Valeria Gigliotti en diversos temas teóricos y sus valiosos consejos. Por último, y no por ello menos importante, agradecer a las críticas y consejos de los evaluadores anónimos que me permitieron ajustar sustancialmente el presente trabajo. La investigación fue realizada mediante el financiamiento parcial del CONICET (Argentina) a través de las becas de posgrado percibidas.

\section{Fuentes primarias}

Husson, F., Josse, J. y Pagès, J. 2010: "Principal component methods - hierarchical clustering - partitional clustering: why would we need to choose for visualizing data?", Technical Report-Agrocampus. [En línea] http://factominer.free.fr/docs/HCPC_husson_josse.pdf [consultado el 10/09/2014].

Martín Hernández, M. 1984: La Tipología en Arquitectura. Tesis doctoral. Universidad de las Palmas de Gran Canaria - Departamento de Arte, Ciudad y Territorio, Las Palmas de Gran Canaria.

Rolón, G. 2013: La vivienda popular riojana del ámbito rural: Patrones arquitectónicos y contexto social en los valles durante el Periodo republicano. Tesis doctoral. Facultad de Filosofía y Letras - Universidad de Buenos Aires.

\section{Bibliografía}

Alexander, C. 1981: El modo intemporal de construir. Gustavo Gilli, Barcelona.

Alexander, C., Ishikawa, S. y Silverstein, M. 1977: A Pattern Language: Towns, Buildings, Construction, vol. 2. Oxford University Press, New York.

Aparicio, F. de 1937: "La vivienda natural en la provincia de La Rioja. Noticia preliminar", Anales de la Sociedad Argentina de Estudios Geográficos, 5, pp. 429-433.

Ardelean, C. 2004: "Factores causales del patrón de asentamiento en arqueología”, Boletín de Antropología Americana, 40, pp. 99-138.

Armellini, O., Cóppola, H., Iglesias Molli, G. y Rosso, R. 1970: “Anexo 3.1. Estudio particularizado de la vivienda en el área", en Programación de viviendas y servicios comunitarios en el Valle Antinaco-Los Colorados: (Provincia de La Rioja), Convenio Consejo Agrario Nacional - Facultad de Arquitectura y Urbanismo de la Universidad de Buenos Aires - Instituto de Investigaciones de la Vivienda.

Ayán, X. 2003: “Arquitectura como tecnología de construcción de la realidad social", Arqueología de la Arquitectura, 2, pp. 17-24.

Ayán, X. 2014: "Todo queda en casa: espacio doméstico, poder y división social en la Edad del Hierro del NW de la Península Ibérica", en S. Gutiérrez Lloret, y I. Grau (eds.), De la estructura doméstica al espacio social. Lecturas arqueológicas del uso social del espacio, pp. 207-222. Publicaciones de la Universidad de Alicante, Alicante.

Azkarate, A. 2002: "Intereses cognoscitivos y praxis social en arqueología de la arquitectura", Arqueología de la Arquitectura, 1, pp. 55-72.

Azkarate, A. 2011: “Archeologia dell'Architettura in Spagna”, Archeologia dell'Architettura, 15, pp. 17-28.
Azkarate, A. 2013: "La construcción y lo construido. Arqueología de la Arquitectura" en J. Quirós Castillo (ed.), La materialidad de la historia. La arqueología en los inicios el siglo XXI, pp. 271-298. Akal, Madrid.

Azkarate, A. y García Gómez, I. 2004: "Las casas-torre bajomedievales. Análisis sistémico de un proceso de reestructuración espacial/territorial", Arqueología de la Arquitectura, 3, pp. 7-37.

Azkarate, A. y Solaun, J. 2012: “Tipologías domésticas y técnicas constructivas en la primitiva Gasteiz (País Vasco) durante los siglos VIII al XII d.C.", Arqueología de la Arquitectura, 9, pp. 103-128.

Barros, N., Thierry, J., Huergo, R. y Alsina, J. 1894: La provincia de La Rioja. Minas. Agricultura. Ganadería. Informe de la Comisión de estudios enviada por el Departamento General de Inmigración en Marzo de 1894. Departamento General de Inmigración, Buenos Aires.

Bermejo Tirado, J. 2009: "Leyendo los espacios: una aproximación crítica a la sintaxis espacial como herramienta de análisis arqueológico", Arqueología de la Arquitectura, 6, pp. 47-62.

Caballero, L. 2009: "Edificio Histórico y Arqueología: un compromiso entre exigencias, responsabilidad y formación", Arqueología de la Arquitectura, 6, pp. 11-19.

Cáceres Freyre, J. 1946: "En torno al estudio de la vivienda rural argentina", Anales de la Asociación Folklórica Argentina, II, pp. 91-93.

Canepuccia, P., Castro, H., Ocvirk, M. y Ostropolsky, E. 1976: Viviendas tradicionales en zona árida: La Rioja, Programa de la Organización de Estados Americanos para la Vivienda, Estudio de la vivienda económica en zonas áridas argentinas. Publicación del Centro de Investigación Mendoza, Mendoza.

Caniggia, G. y Maffei, G.L. 1995: Tipología de la Edificación. Estructura del espacio antrópico. Celeste Ediciones, Madrid.

Chiozza, E. y Aparicio, C de. 1961: "Vivienda rural", en F. Aparicio y H. Difrieri (eds.), La Argentina: Suma de Geografia, tomo 7, pp. 420-562. Ediciones Peuser, Buenos Aires.

Cresta de Suárez, M. y Suárez, M. 1970: "Recursos Hídricos", en H. Lafón, (ed.), Manual de Historia y Geografia de La Rioja, Tomo 2 - Geografía, pp. 201-399. Compañía Editora Riojana, La Rioja.

Criado, F. 1999: "Del Terreno al Espacio: Planteamientos y Perspectivas para la Arqueología del Paisaje", en F. Criado (ed.), CAPA. Criterios y Convenciones en Arqueología del Paisaje, vol. 6, pp. 1-82. Grupo de Investigación en Arqueología del Paisaje, Universidade de Santiago de Compostela, Santiago de Compostela. [En línea] URI: http://hdl.handle. net/10261/5698.

Díaz, R. 1974: La población de la provincia de La Rioja desde el punto de vista geográfico. Editorial La Rioja, La Rioja.

Diez, F. 1996: Buenos Aires y algunas constantes en las transformaciones Urbanas. Editorial de Belgrano, Buenos Aires.

Fuente, A. de la 2007: Los hijos de Facundo: caudillos y montoneras en la provincia de La Rioja durante el proceso de formación del estado nacional argentino: 1853-1870. Prometeo Libros, Buenos Aires.

García Gómez, I. 2009: "Sistemas complejos y arqueología. Una aproximación teórica al fenómeno urbano", Arqueología de la Arquitectura, 6, pp. 63-92.

Güney, Y. 2007: "Type and typology in architectural discourse”, BAÜ FBE Dergisi, 9, 1, pp. 3-18. [En línea] http://fbe.balikesir.edu.tr/dergi/20071/ BAUFBE2007-1-1.pdf [consultado el 10/09/2014].

Gutiérrez Lloret, S. y Grau, I. 2014: De la estructura doméstica al espacio social. Lecturas arqueológicas del uso social del espacio. Publicaciones de la Universidad de Alicante, Alicante.

Hammer, Ø., Harper, D. y Ryan, P. 2001: "PAST: Paleontological Statistics Sofware Package for Education and Data Analysis", Palaeontologia Electronica, 4, 1, pp. 1-9.

Hillier, B. y Leaman, A. 1974: "How is design possible?", Journal of Architectonical Research, 3, 1, pp. 4-11.

Instituto de Investigaciones de la Vivienda 1972: Tipos predominantes de vivienda natural en la República Argentina. Editorial Universitaria de Buenos Aires, Buenos Aires.

Lê, S., Josse, J. y Husson, F. 2008: "FactoMineR: an R package for multivariate analysis", Journal of Statistical Software, 25, 1, pp. 1-18. 
Mañana, P., Ayán, X. y Blanco Rotea, R. 2002: "Bases teórico-metodológicas para una Arqueología de la Arquitectura", en F. Criado (ed.), TAPA. Traballos de Arqueoloxía e Patrimonio, vol. 25, pp. 12-101. Grupo de Investigación en Arqueología del Paisaje, Universidade de Santiago de Compostela, Santiago de Compostela. [En línea] URI: http://hdl.handle. net/10261/6027.

Mountford, M. 1970: "A test of the difference between clusters", en G. Patil, S. Pielou y E. Waters (eds.), Statistical Ecology, pp. 237-257. Pennsylvania State University Press Penna, Estados Unidos.

Navarro Barba, J. 2006: Arquitectura popular provincia de Ávila. Institución Gran Duque de Alba, Ávila.

Olivera, G. 2000: Por travesias y oasis: mercados, producción agraria y actores sociales de la Rioja (Los Llanos y Arauco, 1900-60). Editorial Universidad Nacional de Córdoba, Córdoba.

Olivera, G. 2001a: “Articulación mercantil y transformaciones sociales agrarias en Los Llanos (La Rioja, 1900-1960)", en S. Bandieri (coord.), Cruzando la Cordillera. La Frontera Argentino-Chilena como Espacio Social, pp. 279-319. Universidad Nacional de Comahue, Neuquén.

Olivera, G. 2001b: “Olivo, políticas sustitutivas y heterogeneidad agraria: (La Rioja 1940-1970)”, Mundo agrario, 1, 2. [En línea] http://www.redalyc. org/pdf/845/84510204.pdf [consultado el 10/09/2014]

Pagès, J. 2004: “Analyse factorielle de données mixtes", Revue de statistique appliquée, 54, 4, pp. 93-111.

Pastor, G. 2000: "Vivienda vernácula del noroeste argentino el caso de la vivienda rural de Tucumán. Siete aspectos para una definición de la vivienda rural del Valle de Tafi', Gazeta de antropología, 16, artículo 25. [En línea] URI: http://hdl.handle.net/10481/7520.

Plata, A. 2003: "La aplicación de la arqueología de la arquitectura a un complejo productivo. El valle salado de Salinas de Añana (Álava)", Arqueología de la Arquitectura, 2, pp. 241-248.

Pugh, T. 2003: "A cluster and spatial analysis of ceremonial architecture at Late Postclassic Mayapán”, Journal of Archaeological Science, 30, pp. 941-953.
Rolón, G. y Rotondaro, R. 2010: "Empleo del método estratigráfico en el estudio de la vivienda rural vernácula construida con tierra: un caso de aplicación en La Rioja, Argentina”, Arqueología de la Arquitectura, 7, pp. 213-222.

Rolón, G. y Rotondaro, R. 2011: "El agua de riego en la construcción del paisaje cultural en las zonas áridas del centro-oeste argentino: Un caso de estudio: Chañarmuyo, La Rioja", Revista de geografia Norte Grande, 48, pp. 159-177.

Rossi, A. 1977: Para una arquitectura de tendencia: escritos 1956-1972. Gustavo Gili, Barcelona.

Sánchez, M. 1928: La provincia de La Rioja. Estudio físico, político y económico. Talleres Gráficos Argentinos L. J. Rosso, Buenos Aires.

Sánchez Zufiaurre, L. 2007: Técnicas constructivas medievales: nuevos documentos arqueológicos para el estudio de la Alta Edad Media en Álava. Servicio Editorial de la Universidad del País Vasco, Leioa.

Sneath, P. y Sokal, R. 1973: Numerical taxonomy. W. H. Freeman and Company, San Francisco.

Tomasi, J. y Rivet, C. 2011: "Que el barro esté bien liviano. El torteado con barro en los techos de Susques y Rinconada, provincia de Jujuy", en J. Tomasi, y C. Rivet (eds.), Puna y arquitectura. Las formas locales de la construcción, pp. 113-123. Centro de Documentación de Arte y Arquitectura Latinoamericana, Buenos Aires.

Van Dyke, R. 1999: "The Chaco Connection: Evaluating Bonito-Style Architecture in Outlier Communities", Journal of Anthropological Archaeology, 18, pp. 471-506.

Vargas Lorenzo, C. 2013: "Reflexiones sobre cronotipologías en Arqueología de la Arquitectura", Arqueología de la Arquitectura, 10, e001. [En línea] doi: http://dx.doi.org/10.3989/arq.arqt.2013.001.

Vigil-Escalera, A. 2014: "Espacio social y espacio doméstico en los asentamientos campesinos del Centro y Norte peninsular (siglos V-IX d.c.)", en Gutiérrez Lloret, S. y Grau, I. (eds.), De la estructura doméstica al espacio social. Lecturas arqueológicas del uso social del espacio, pp. 207-222. Publicaciones de la Universidad de Alicante, Alicante.

Viñuales, G. 1991: "La arquitectura en tierra en la región andina", Anales del Instituto de Arte Americano e Investigaciones Estéticas, 27-28, pp. 43-53. 